\title{
A review of optimization techniques in spacecraft flight trajectory design
}

\author{
Runqi Chai ${ }^{\mathrm{a}, *}, \mathrm{Al}$ Savvaris $^{\mathrm{a}}$, Antonios Tsourdos ${ }^{\mathrm{a}}$, Senchun Chai ${ }^{\mathrm{b}}$, Yuanqing \\ $\mathrm{Xia}^{\mathrm{b}}$ \\ ${ }^{a}$ School of Aerospace, Transport and Manufacturing, Cranfield University, Bedfordshire, \\ MK43 OAL, United Kingdom \\ ${ }^{b}$ School of Automation, Beijing Institute of Technology, Beijing 100081, PR China
}

\begin{abstract}
For most atmospheric or exo-atmospheric spacecraft flight scenarios, a welldesigned trajectory is usually a key for stable flight and for improved guidance and control of the vehicle. Although extensive research work has been carried out on the design of spacecraft trajectories for different mission profiles and many effective tools were successfully developed for optimizing the flight path, it is only in the recent five years that there has been a growing interest in planning the flight trajectories with the consideration of multiple mission objectives and various model errors/uncertainties. It is worth noting that in many practical spacecraft guidance, navigation and control systems, multiple performance indices and different types of uncertainties must frequently be considered during the path planning phase. As a result, these requirements bring the development of multi-objective spacecraft trajectory optimization methods as well as stochastic spacecraft trajectory optimization algorithms. This paper aims to broadly review the state-of-the-art development in numerical multi-objective trajectory optimization algorithms and stochastic trajectory planning techniques for spacecraft flight operations. A brief description of the mathematical formulation of the problem is firstly introduced. Following that, various optimization methods that can be effective for solving spacecraft trajectory planning problems are reviewed, including the gradient-based methods, the convexification-based methods, and the evolutionary/metaheuristic methods. The multi-objective
\end{abstract}

\footnotetext{
${ }^{*}$ Corresponding author

Email addresses: r.chai@cranfield.ac.uk (Runqi Chai), a.savvaris@cranfield.ac.uk (Al Savvaris), a.tsourdos@cranfield.ac.uk (Antonios Tsourdos), chaisc97@bit.edu.cn (Senchun Chai), xia_yuanqing@bit.edu.cn (Yuanqing Xia)
} 
spacecraft trajectory optimization formulation, together with different class of multi-objective optimization algorithms, is then overviewed. The key features such as the advantages and disadvantages of these recently-developed multiobjective techniques are summarised. Moreover, attentions are given to extend the original deterministic problem to a stochastic version. Some robust optimization strategies are also outlined to deal with the stochastic trajectory planning formulation. In addition, a special focus will be given on the recent applications of the optimized trajectory. Finally, some conclusions are drawn and future research on the development of multi-objective and stochastic trajectory optimization techniques is discussed.

Keywords: Flight trajectories, Multi-objective spacecraft trajectory optimization, Stochastic spacecraft trajectory optimization, Robust optimization strategies.

\section{Nomenclature}

\begin{tabular}{|c|c|c|}
\hline NLP & $=$ & nonlinear programming \\
\hline FONC & $=$ & first order necessary condition \\
\hline SQP & $=$ & sequential quadratic programming \\
\hline IP & $=$ & interior point \\
\hline IPSQP & $=$ & interior point sequential quadratic programming \\
\hline LP & $=$ & linear programming \\
\hline $\mathrm{SOCP}$ & $=$ & second order cone programming \\
\hline SDP & $=$ & semidefinite programming \\
\hline DP & $=$ & dynamic programming \\
\hline DDP & $=$ & differential dynamic programming \\
\hline SDDP & $=$ & stochastic differential dynamic programming \\
\hline GA & $=$ & genetic algorithm \\
\hline $\mathrm{DE}$ & $=$ & differential evolution \\
\hline VLDE & $=$ & violation learning differential evolution \\
\hline PSO & $=$ & particle swarm optimization \\
\hline PPPIO & $=$ & predator-prey pigeon-inspired optimization \\
\hline $\mathrm{AC}$ & $=$ & ant colony \\
\hline $\mathrm{ABC}$ & $=$ & artificial bee colony \\
\hline SA & $=$ & simulate annealing \\
\hline
\end{tabular}




\begin{tabular}{|c|c|c|}
\hline $\mathrm{TS}$ & $=$ & tabu search \\
\hline MOTO & $=$ & multi-objective trajectory optimization \\
\hline NSGA-II & $=$ & nondominated sorting genetic algorithm II \\
\hline I-NSGA-II & $=$ & improved nondominated sorting genetic algorithm II \\
\hline NSGA-III & $=$ & nondominated sorting genetic algorithm III \\
\hline $\mathrm{MOEA} / \mathrm{D}$ & $=$ & multi-objective evolutionary algorithm Based on decomposition \\
\hline MOPSO & $=$ & multi-objective particle swarm optimization \\
\hline MOAPSO & $=$ & multi-objective adaptive particle swarm optimization \\
\hline MOAGPSO & $=$ & multi-objective adaptive gradient particle swarm optimization \\
\hline MOABC & $=$ & multi-objective artificial bee colony \\
\hline NPGA & $=$ & niched pareto genetic algorithm \\
\hline SPPSO & $=$ & strength pareto particle swarm optimization \\
\hline ADEMGT & $=$ & adaptive differential evolution and modified game theory \\
\hline WS & $=$ & weighted-sum \\
\hline $\mathrm{PP}$ & $=$ & physical programming \\
\hline FPP & $=$ & fuzzy physical programming \\
\hline IFPP & $=$ & interactive fuzzy physical programming \\
\hline GP & $=$ & goal programming \\
\hline FGP & $=$ & fuzzy goal programming \\
\hline FSGP & $=$ & fuzzy satisfactory goal programming \\
\hline $\mathrm{ASM}$ & $=$ & adaptive surrogate model \\
\hline EMO & $=$ & evolutionary multi-objective optimization \\
\hline MOEA & $=$ & multi-objective evolutionary algorithm \\
\hline MOT & $=$ & multi-objective transcription \\
\hline SOP & $=$ & single-objective problem \\
\hline $\mathrm{CC}$ & $=$ & chance constraint \\
\hline $\mathrm{RO}$ & $=$ & robust optimization \\
\hline $\mathrm{CCO}$ & $=$ & chance-constrained optimization \\
\hline $\mathrm{SDE}$ & $=$ & stochastic differential equation \\
\hline $\mathrm{SQF}$ & $=$ & stochastic quadrature formula \\
\hline $\mathrm{PDF}$ & $=$ & probability density function \\
\hline $\mathrm{gPC}$ & $=$ & generalized polynomial chaos \\
\hline MCMC & $=$ & Markov chain Monto Carlo \\
\hline$x$ & $=$ & state variable \\
\hline$u$ & $=$ & control variable \\
\hline
\end{tabular}




$$
\begin{aligned}
t & =\text { time } \\
b & =\text { boundary function } \\
g & =\text { path function } \\
J & =\text { objective function } \\
\Phi & =\text { Mayer cost } \\
L & =\text { process cost } \\
E & =\text { number of equality constraints } \\
I & =\text { number of inequality constraints } \\
M & =\text { number of objective functions } \\
\xi & =\text { uncertain parameter } \\
\epsilon & =\text { acceptable probability of occurrence } \\
w_{k} & =\text { weighted parameter }
\end{aligned}
$$

\section{Introduction}

In the last couple of decades, numerous achievements and massive efforts have been witnessed in order to move human beings into space. Nowadays, aerospace science and technology has brought various changes in not only the military field but also scientific and engineering applications. Among them, the development of spacecraft technology has attracted significant attention $[1,2]$. So far, several generations of spacecraft have been designed, manufactured, launched, and successfully implemented in different mission profiles such 15 as communications [3], interplanetary travel [4], regional reconnaissance [5], environmental monitoring [6], and so on. Because of the long development cycle, high operating cost, and limited resources, it is usually desired by aerospace engineers that the space vehicle can fulfill the mission with some performance metrics to be optimized, or in other words, in an optimal or near-optimal way.

20 To achieve this goal, a proper treatment of the flight trajectory for the space vehicle is often required, and this stimulates the development of trajectory optimization techniques.

It has been shown in many published works that the trajectory design component plays a key role with regard to stable flight and improved control the use of trajectory optimization in different space missions, together with various related trajectory optimization approaches, was made by Conway in 
2011 [2]. In this review article and the references therein, several important practical examples were highlighted such as the orbital transfer problems $[9$, 10], the spacecraft rendezvous and docking [11, 12], and the planetary entry [13-17]. These problems were summarised in a general form and treated as optimal control problems [18]. It is worth noting that according to Betts [1], an interchanged designation between the term "optimal control problems" and "trajectory optimization problems" can always be found in the literature. A 35 rigours analysis of the differences between these two statements is beyond the scope of this survey. Readers are referred to [19] and [20] for a more complete description and analysis of these differences.

From the current development of optimal control theory on the whole, the development/application of numerical trajectory optimization methods for atmospheric or exo-atmospheric spacecraft flight scenarios leads to two different trends. The first one is that system discretization tends to become more reliable and adaptive such that it can maximally capture the characteristics of the dynamical system $[18,21]$. The other is that optimization becomes more accurate and computationally friendly so that the solution optimality, together

45 with the real-time capability, can be improved. Depending on the order of discretization and optimization, numerical trajectory optimization methods can be classified into two main categories. That is, the so-called indirect methods ("optimization then discretization") and the direct methods ("discretization then optimization") [21]. The former type of method aims to solve the first-order 50 necessary conditions for optimality with respect to the spacecraft trajectory optimization problems. Successful examples have been reported in the literature for addressing problems without considering inequality constraints [22, 23]. In these works, the first-order necessary conditions were formulated as two-point boundary value differential-algebraic equations. However, in terms of problems ${ }_{55}$ in the presence of inequality constraints, this type of approach might not be effective. This is because it is difficult to determine the switch points where the inequality constraints become active, thus limiting the practical application of this type of method. As for the direct method, the first step is to discretize the control or the state and control variables so as to transform the original formulation to a static nonlinear programming problem (NLP). Following that, different well-developed optimization techniques are available to address the optimal solution of the resulting static problem. Compared with the indirect 
strategy, it is much easier to apply the direct method to handle the spacecraft trajectory design problem. Moreover, the way of formulating constraints tends to be more straightforward. Therefore, applying the "discretization then optimization" mode has attracted more attention in engineering practice.

The primary goal of this review article is to present the latest progress that has been achieved in the development of spacecraft trajectory optimization techniques. Specifically, the main focus will be on the recently-proposed optimization methods that have been utilized in constrained trajectory optimization problems, multi-objective trajectory optimization problems, and stochastic trajectory optimization problems. Therefore, compared with the optimization process, the discretization process is less important and will only be briefly mentioned in the following sections. A detailed and serious attempt to clas-

75 sify discretization techniques for spacecraft trajectory design can be found in $[10,20]$.

One individual objective of this work is to summarise the main advantages and disadvantages of applying different optimization methods in spacecraft trajectory optimization problems based on the results reported in the newly-published works. It should be noted that significant differences might be found when applying one optimization strategy, which can achieve promising results for a specific mission, to a different task. This phenomenon becomes even apparent when the problem is extended to the multi-objective version and the stochastic version. However, thanks to the pioneering works carried out by many aerospace researchers, a large number of competitive results of various benchmark problems are now available. Consequently, it is possible to gain a better understanding about how these different optimization algorithms behave in dealing with complex mission scenarios.

The rest of this survey will be organized as follows: Section 2 provides a brief description of the mathematical formulation of the spacecraft trajectory optimization problem. Subsequently, different types of optimization methods that can be effective for addressing the problem are summarised in Section 3. These algorithms include the gradient-based methods, the evolutionary-based (i.e., heurestic/metaheuristic) methods, the convexification-based methods, and 95 the dynamic programming-based methods. The multi-objective spacecraft trajectory optimization formulation, along with various classes of multi-objective optimization algorithms, will then be reviewed in Section 4. The key features 
such as the advantages and disadvantages of applying these newly-developed multi-objective trajectory planning techniques are also discussed. Section 5

\subsection{Continuous Dynamical Systems}

Currently, numerous dynamical systems exist in the literature that are applied to describe the movement of space vehicles for different missions. Although differences can be found in terms of the mission profiles and the type of vehicle, many of the examples are able to be summarised into a general form. For 

ment of the $N_{x}$-dimensional state variable $x \in \mathbb{R}^{N_{x}}$, which can be written as [24]:

$$
\dot{x}(t)=f(x(t), u(t), t)
$$

where $f$ is a function with respect to time $t \in \mathbb{R}$, the state $x \in \mathbb{R}^{N_{x}}$ and control $u \in \mathbb{R}^{N_{u}}$ variables. $f: \mathbb{R}^{N_{x}} \times \mathbb{R}^{N_{u}} \times \mathbb{R} \mapsto \mathbb{R}^{n_{x}}$ is usually nonlinear. Usually, the state trajectory $x(t)$ begins at a given point $x_{0}$ at a given time instant $t_{0}$, and some state variables are required to terminate at a pre-specified final point $x_{f}$ at the final time instant $t_{f}$. This boundary condition can be summarised to an inequality, which has the form of:

$$
b_{L} \leq b\left(x_{0}, t_{0}, x_{f}, t_{f}\right) \leq b_{U}
$$

where $b_{L}, b_{U} \in \mathbb{R}^{N_{b}}$ are the lower and upper bounds of $b(\cdot, \cdot, \cdot, \cdot)$, respectively. $b: \mathbb{R}^{N_{x}} \times \mathbb{R} \times \mathbb{R}^{N_{x}} \times \mathbb{R} \mapsto \mathbb{R}^{N_{b}}$.

\subsection{Variable/Path Constraints}

Apart from the consideration of the vehicle dynamical system, various variable/path constraints should be also taken into account so as to fulfill the mission-dependent requirements or to protect the structural integrity of the vehicle. These requirements are usually named path constraints and they can be modeled in a general form (i.e., a general inequality with lower and upper bounds):

$$
g_{L} \leq g(x(t), u(t), t) \leq g_{U}
$$

where $g_{L}, g_{U} \in \mathbb{R}^{N_{g}}$ are the lower and upper bounds of the path function $g(\cdot, \cdot, \cdot)$, respectively. $g: \mathbb{R}^{N_{x}} \times \mathbb{R}^{N_{u}} \times \mathbb{R} \mapsto \mathbb{R}^{N_{g}}$.

It should be noted that for different spacecraft flight mission profiles, there exist various path constraints. For instance, in [25], a reusable launch vehicle trajectory optimization model was constructed. In this problem, three flight path constraints including the aerodynamic heating, dynamic pressure and load factor were required to be satisfied during the optimization process. Similarly, considering the no-fly zone constraints that limit the lateral and longitudinal positions of the vehicle, a three-degree-of-freedom spacecraft reentry model was established and solved in [26]. In addition, in [27] a spacecraft rendezvous and docking problem was considered. In this work, in order to achieve the thrust direction limitations, a path constraint was imposed on the control variables. 
Nevertheless, all these constraints can be classified into the above two types

155 as indicated by Eq.(2). It is obvious from Eq.(2) that the path constraints are required to be satisfied during the entire time domain (e.g. for $\forall t \in\left[t_{0}, t_{f}\right]$ ), and they can be pure control path constraints (i.e., functions of control variables) [28], mixed path constraints (i.e., functions of state and control variables) [29], or pure state constraints (i.e., functions of state variables) [30].

\subsection{Mission Objectives}

A solution to the space vehicle dynamical system (1) which satisfies all the path constraints can only be recognized as a feasible flight trajectory. Usually, for a given mission profile, a large amount of feasible flight trajectories can be found. The selection of the particular one among the feasible set is based on a suitably defined performance index that quantifies the magnitude of goal attainment specified by the designers. Commonly, the mission objective (cost function) to be optimized can be defined in the following Bolza form:

$$
J=\Phi\left(x_{0}, t_{0}, x_{f}, t_{f}\right)+\int_{t_{0}}^{t_{f}} L(x(t), u(t), t) d t
$$

From Eq.(3), the cost function is composed of two parts. The term $\Phi$ : $\mathbb{R}^{N_{x}} \times \mathbb{R} \times \mathbb{R}^{N_{x}} \times \mathbb{R} \mapsto \mathbb{R}$ denotes the Mayer cost, whereas $L: \mathbb{R}^{N_{x}} \times \mathbb{R}^{N_{u}} \times \mathbb{R} \mapsto$ $\mathbb{R}$ stands for the process cost. Similarly with the path constraint case, the definitions of performance index may vary from mission to mission but most of them can be written as the general form given by Eq.(3). For instance, for the spacecraft reentry mission considered in [31], the primary task is to maximize the final latitude, thus leading to a larger cross range value. Other potential objectives for this mission are minimizing the total amount of aerodynamic heating [16], maximizing the final kinetic energy [32], minimizing the smoothness of the obtained flight path and so on [33]. Furthermore, a low-thrust interplanetary mission was studied in [34], wherein maximizing the delivered mass was chosen as the main objective. Besides, in [10] the authors considered a multiple-pass orbital transfer problem for the aeroassisted spacecraft. As the mission might contain several subsegments, minimizing the fuel consumption during the transfer (mass fraction) was selected as the main objective.

It should be noted that in Eq.(3), not only the final time cost but also the initial time cost is included in the Mayer cost term $\Phi$. Actually, this is critical for some missions such as the launch vehicle applications or orbital transfer 
problems where the initial values of the state and time must be optimized [35, $36]$.

\subsection{Overall Formulation}

Based on the introduction of the vehicle dynamics, different types of conproblems is displayed in Fig.1.

\subsection{Numerical Solution Approach}

As discussed in the introduction section of this article, there are two commonly used strategies, named indirect methods and direct methods, for addressing the spacecraft trajectory optimization problems. Specifically, in the former class, it is required to derive the first order necessary conditions (FONCs) for optimality via the calculus of variations. Subsequently, the original optimal control formulation is transformed to a Hamiltonian boundary-value problem, thereby resulting in an "optimization + discretization" solution-finding structure. In the later class, a "discretization + optimization" strategy is adopted. 205 That is, certain parametrization methods are firstly applied to transform the continuous-time system to a static version. Following that, the original problem formulation is rewritten as a static nonlinear programming problem and welldeveloped optimization algorithms can be used to produce the optimal solution. A graphical illustration of numerical solution approaches that were developed in the past two decades for the solution of spacecraft trajectory optimization 


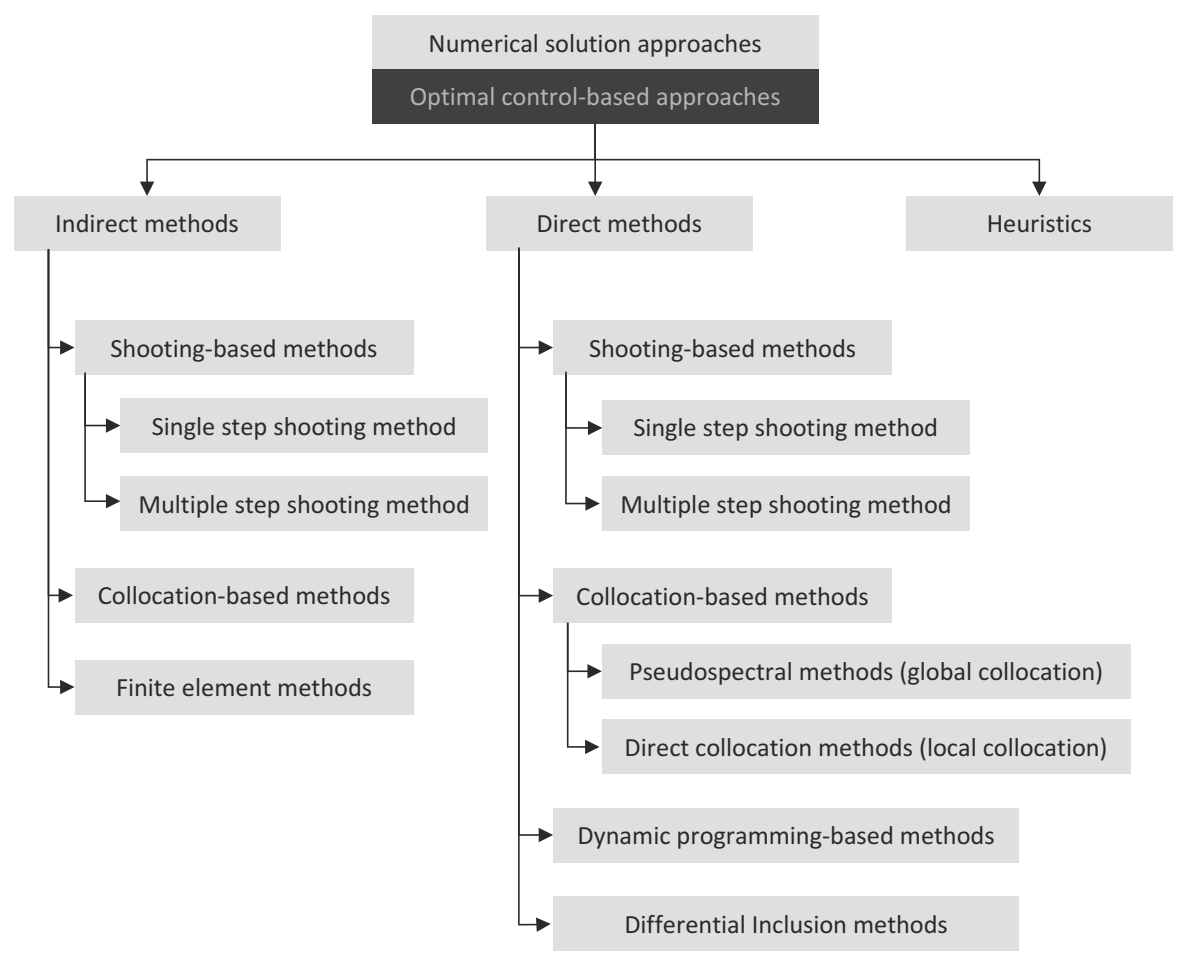

Figure 1: Numerical solution approaches

It is worth noting that in an indirect method, the construction of the FONCs usually becomes costly due to the complexity of the vehicle dynamics and various constraints. In addition, the number of the resulting decision variables associated with this method is large. Therefore, from an implementation point of view, the second class of strategy (i.e., direct methods) may attract more attention. In this paper, we are interested in reviewing the latest developments of optimization techniques that are effective and reliable to generate the optimal spacecraft flight trajectories. Detailed introduction and analysis of different discretization techniques is beyond the scope of this research. We refer to [20] for such a broad review.

It is worth noting that there are also some other geometric-based trajectory planning approaches that have been reported in the literature [37-39]. These geometric-based algorithms can have acceptable performance for producing feasible trajectories and they are mainly used as the motion planners for the 
aircraft, UAVs, or autonomous ground vehicles. However, since the main focus of this work is to review trajectory optimization approaches related to the space vehicle literature, the geometric-based algorithm is removed from the algorithm tree shown in Fig.1.

\section{Optimization Algorithms}

As indicated in the previous section, to solve the spacecraft trajectory design problem, an important procedure is to apply optimization methods to solve the static nonlinear programming problem (NLP). A standard NLP problem can be written as [40]:

$$
\begin{array}{ll}
\text { Find decision variables } & x=\left[x_{1}, x_{2}, \ldots, x_{n}\right] \\
\text { Minimize objective function } & J(x) \\
\text { subject to } & x_{\min } \leq x \leq x_{\max } \\
& H_{i}(x)=0 \\
& G_{j}(x) \leq 0 \\
& (i=1,2, \ldots, E) \\
& (j=1,2, \ldots, I)
\end{array}
$$

where $E$ and $I$ are the dimensionality of the equality and inequality constraints, respectively. Currently, there are many effective optimization techniques that can be applied to solve the NLPs.

The main objective of this section is to review the state-of-the-art optimization strategies reported in the literature for calculating the optimal spacecraft 240 flight trajectories. Based on the reported results, one may be able to gain a better understanding in terms of the performance and behaviours of different algorithms for addressing various space vehicle flight missions. Moreover, it is possible to guide the reader to improve one of these techniques in order to circumvent the limitations brought by the classic methods.

In the literature, four types of optimization strategies are usually applied to solve the spacecraft trajectory optimization problems. Specifically, the gradientbased, convexification-based, dynamic programming-based, and derivative-free (heuristic-based) optimization techniques are used to calculate the optimal time history with respect to the spacecraft state and control variables. These algorithms can be further grouped into the deterministic and the stochastic approaches, and the most popular optimization methods among these two groups 
are summarised and tabulated in Table 1 and Table 2. It should be noted that not all the optimization algorithms under each category are listed in this table. Alternatively, only some important examples are reviewed and these techniques are discussed in detail in the following subsections. A large number of numerical simulations were carried out in related works. The results indicated that these newly-proposed optimization strategies are effective and can provide feasible solutions for solving the constrained space vehicle trajectory design problems.

Table 1: Popular deterministic optimization algorithms available for trajectory optimization problems

\begin{tabular}{l}
\hline \hline Deterministic Optimization Algorithms \\
\hline Sequential quadratic programming (SQP) [9] \\
Interior point method (IP) [41] \\
Interior point sequential quadratic programming (IPSQP) [42] \\
Linear programming (LP) [43] \\
Second order cone programming (SOCP) [44] \\
Semidefinite programming (SDP) [45] \\
Dynamic programming (DP) [46] \\
Differential dynamic programming (DDP) [47] \\
Stochastic differential dynamic programming (SDDP) [48] \\
\hline \hline
\end{tabular}

Table 2: Popular stochastic optimization algorithms available for trajectory optimization problems

\begin{tabular}{l}
\hline \hline Stochastic Optimization Algorithms \\
\hline Genetic algorithm (GA) [13] \\
Differential evolution (DE) [49] \\
Violation learning differential evolution (VLDE) [50] \\
Particle swarm optimization (PSO) [12] \\
Predator-prey pigeon-inspired optimization (PPPIO) [51] \\
Ant colony (AC) [52] \\
Artificial bee colony (ABC) [53] \\
Simulate annealing (SA) [54] \\
Tabu search (TS) [55] \\
\hline \hline
\end{tabular}




\subsection{Gradient-based Methods}

the spacecraft flight trajectory is the classic gradient-based method. Among gradient-based methods, the sequential quadratic programming (SQP) method and the interior point (IP) method are used successfully for the solution of large scale NLP problems [56]. In [57], a fuel-optimal aeroassisted spacecraft orbital discretization method. Then, the static NLP was solved by applying the standard SQP method to generate the fuel-optimal flight trajectory. Similarly, in [9] the SQP method was applied as the primary optimizer to search the timeoptimal flight trajectory of a low-thrust orbital transfer problem. Generally, the aim for the SQP algorithm is to transform the original problem to a sequence of quadratic programming subproblems by approximating the augmented Lagrangian quadratically and linearizing the constraints using Taylor expansion. More precisely, each Newton iteration of the SQP loop requires the solution of a quadratic programming subproblem containing Jacobian and Hessian matrix.

275 The solution-finding steps of SQP method can be summarised as the following steps:

Step 1 Construct the augmented Lagrangian function.

Step 2 Apply the quadratic model to approximate the augmented Lagrangian.

Step 3 Input the initial guess value $x_{k}$.

Step 4 Use the Newton method to calculate the step direction $g_{k}$.

Step 5 Calculate the step length $\alpha_{k}$ based on the sufficient decrease conditions.

Step 6 Check the stopping optimality tolerance $\epsilon$ of the current solution.

Step 7 If the stopping condition cannot be satisfied.

Step 8 Update $x_{k+1}=x_{k}+\alpha_{k} d_{k}$.

Step 9 Set $k=k+1$ and go back to Step 4 .

Step 10 If the stopping condition can be satisfied.

Step 11 Terminate the algorithm and output the optimal solution. 


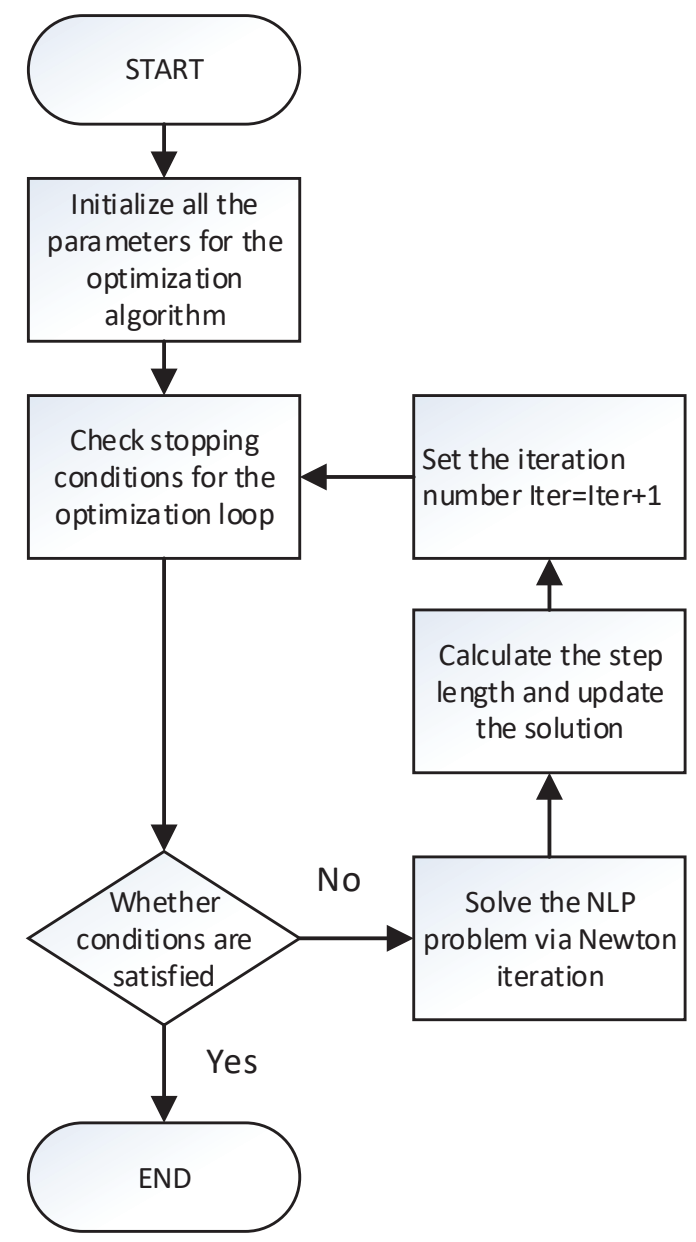

Figure 2: General steps of using gradient-based method

A graphical illustration about the general steps of using gradient-based methods to solve problem (5) is depicted in Fig.2.

It should be noted that when solving the optimization problem, a line search algorithm is usually a key for enhanced robustness of the algorithm (as indicated in Step 4 to Step 9, and Fig.2). Although there exist a variety of line search strategies (interested readers are referred to Chapter 3 in [40] for more details), they all share a similar philosophy. That is, at each solution-finding iteration $k$, a search direction $d_{k}$ is firstly produced via the Newton, quasi-Newton, or gradient directions. Subsequently, the step length $\alpha_{k}$ is determined along the 
pre-specified searching direction. To select a proper $\alpha_{k}$ such that the objective can have a sufficient improvement, some conditions can then be applied such as the Armijo condition, the Wolfe condition, and the Goldstein condition [40, 42].

Although SQP methods can be used as an effective algorithm to produce the optimal flight trajectory, most of the SQP implementations require the exact solution of the subproblem. This may increase the computational burden of the solver significantly [42]. Moreover, since most SOP methods utilize the active set strategy to handle inequality constraints, the computational burden may be increased if the active set is initialized in an improper way.

Apart from the SQP method, an alternative gradient-based method is the interior point (IP) method developed during the last decade. Investigations of IP can be found in a large amount of work. To apply this method, the inequality constraints need to be transcribed to equality constraints by introducing some slack variables such that the problem can be solved in a simpler form. An application of the IP method in space vehicle trajectory design problem can be found in [41]. In this work, a space shuttle atmospheric reentry problem was considered and discretized via a shooting method. The resulting static NLP problem was then addressed by applying the IP method. Simulation results provided in this work confirmed the effectiveness of applying the IP method. However, it is worth noting that for the IP method, a main challenge is to define the penalty functions and initialize the penalty factor in the augmented merit function in order to measure the quality of the optimization process.

In [42], combining the advantages of the SQP and IP methods, the authors proposed a two-nested gradient-based method, named interior point sequential quadratic programming (IPSQP), for solving the aeroassisted spacecraft trajectory design problem. One important feature of this approach is that an inner solution finding loop was embedded in the algorithm framework, thereby allowing the QP subproblem to be solved inexactly. In this way, the design can 325 have more flexibility to control the optimization process and the algorithm efficiency can also be improved to some extent. Simulation results and comparative studies were reported to show the effectiveness as well as the reliability of this improved gradient-based method.

\subsection{Evolutionary-based Methods}

330 In an optimization problem, if it is hard to get the gradient information of the objective functions or constraints (i.e., due to the high nonlinearity involved 
in these functions), the classic gradient-based method might no longer be reliable or available. In this case, the evolutionary-based methods, also known as global optimization methods, become the only way to produce the optimal solution, as there is no derivative information required in an evolutionary approach. This indicates that it will not suffer from the difficulty of calculating the Jacobian as well as the Hessian matrix.

Evolutionary algorithms or global optimization methods use the principle of "survival of the fittest" adopted to a population of elements representing candidate solutions $[2,15,58]$. Compared with classic gradient-based algorithms, there is no initial guess value required by the algorithms as the population is initialized randomly. Thanks to the nature of the evolutionary algorithm, it tends to be more likely than classic gradient methods to locate the global minimum $[12]$.

There are many types of evolutionary algorithms that are available to produce the optimal solution of an engineering optimization problem. For example, the generic class of evolutionary algorithms such as the genetic algorithm (GA) and differential evolution (DE), the agent-based class such as the particle swarm optimization (PSO) and the pigeon-inspired optimization (PIO), and the colony-based class of algorithms such as the ant colony optimization (ACO) and the artificial bee colony (ABC) algorithm. Relative works on developing or applying these global optimization methods in spacecraft trajectory design are widely researched in the literature. In [59], a constrained space capsule reentry trajectory design problem was addressed by applying a modified GA. Similarly, Kamesh et al. [60] incorporated a hybrid GA and a collocation method so as to address an Earth-Mars orbital transfer task. The authors in [58] produced the optimal path for a space robotic manipulator by using a standard PSO method.

Conway et al. [2] combined global optimization algorithms with standard gradient-based method in order to construct a bi-level structural optimal control method. In their latest work, Pontani and Conway [12] utilized a modified particle swarm optimization algorithm to globally optimize the flight path of a cycling spacecraft.

An enhanced differential evolution approach incorporated with a violationdegree based constraint handling strategy was constructed in our previous work to approximate the optimal flight trajectory [50] of a space maneuver vehicle entry problem. In this work, a simplex-based direct search mechanism was 
embedded in the algorithm framework in order to improve the diversity of the current population. Besides, a learning strategy was used to avoid the premature convergence of the algorithm.

Furthermore, the authors in [61] established an ant colony inspired optimization algorithm so as to plan a multi-phase space vehicle orbital flight trajectory. An automated approach based on genetic algorithm and monotonic basin hopping was applied in [62] to address a launch vehicle interplanetary trajectory problem.

Although the aforementioned works have shown the feasibility of using heuristic-based methods for addressing spacecraft trajectory design problems, the validation of solution optimality becomes difficult. Moreover, the computational complexity due to the heuristic optimization process tends to be very high [63]. Therefore, it is still difficult to treat heuristic-based methods as a "standard" optimization algorithm that can be applied to solve general spacecraft trajectory planning problems. Much effort is expected to improve the computational performance of this kind of algorithm.

\subsection{Convexification-based Methods}

Recently, a growing interest can be found in applying convexification-based

methods for generating the optimal spacecraft flight trajectories [64]. An important feature of applying this kind of method is that it can be implemented with theoretical guarantees with regard to the solution and computational efficiency. Since most of the practical spacecraft trajectory optimization problems are usually nonconvex, in order to apply convex optimization method, various convexification techniques are developed to transform the original problem formulation to a convex version. This can also be understood as using a specific convex optimization model to approximate the original nonconvex formulation. Commonly, there are three types of convex optimization existing in the literature:

395 1. Linear programming model (LP).

2. Second-order cone programming model (SOCP)

3. Semi-definite programming model (SDP)

In terms of the LP model, it should be noted that if the considered problem is relatively complex (i.e., the nonlinearity of the system dynamics, objectives 400 or constraints is high), then the LP model might not be sufficient and reliable 
to approximate the original problem formulation. On the other hand, as for the SDP model, although it has the most accurate approximation ability among the three models listed above, the transformed convex formulation is often not well-scaled, thereby resulting in an increase with regards to the computational racy and the computational complexity can be achieved by applying the SOCP model. This strategy approximates the problem constraints using the second order cone such that the transformed problem can be solved with a relativelysmall computing power.

410 ods to solve space vehicle trajectory design problems can be found in the literature. For example, in $[65,66]$, the planetary landing problem was addressed by using the convex optimization method under the consideration of nonconvex thrust magnitude constraints. Also, in [67], the SOCP method was applied to produce the optimal trajectory of the spacecraft entry planning problem. In this work, nonconvex collision-avoidance constraints as well as the navigation uncertainties were also taken into account and reformulated into convex constraints during the optimization phase.

\subsection{Dynamic Programming-based Methods}

420 on their enhanced ability in achieving stable performance and in dealing with local optimal solution, that naturally exist in nonlinear optimal control problems. In this subsection, two typical dynamic programming-based algorithms are reviewed such as the standard dynamic programming (DP) method, and the differential dynamic programming method (DDP).

Motivated by the Bellman's principle of optimality, DP is proposed and applied to solve engineering optimization problems [46]. The primary idea of the Bellman's principle is that the optimal solution will not diverge if other points on the original optimal solution are chosen as the starting point to re${ }_{430}$ trigger the optimization process. Based on this principle, DP calculates the optimal solution for every possible decision variable. Hence, it is highly likely to result in the curse of dimensionality [48].

In order to deal with the main deficiency faced by the standard DP, the DDP approach has been designed [68]. In this method, the solution-finding 
process is performed locally in a small neighbourhood of a reference trajectory. Subsequently, this method calculates the local optimal solution by using a backward and a forward sweep repeatedly until the solution converges. The DDP method has been successfully applied to calculate the optimal solution of some space missions. For example, in [69, 70], a comprehensive theoretical development of the DDP method, along with some practical implementation and numerical evaluation was provided. In [68], a DDP-based optimization strategy was proposed and applied to calculate the rendezvous trajectory to near Earth objects.

However, most of the recent DDP work does not take the model uncertainties and noises into account in the process of finding the solution. Consequently, the solution finding process might fail to produce a nominal solution which can guarantee the feasibility all along the trajectory when uncertainties or model errors perturb the current solution.

According to all the relative works reported, it can be concluded that although the results generated from most existing optimization algorithms can be accepted as near-optimal solutions, there is still room for improvement with respect to applying these optimization strategies in spacecraft trajectory design problems.

\section{Multi-Objective Spacecraft Trajectory Optimization}

In the past, early investigations on space vehicle trajectory design problems usually focused on one single objective, for example, minimizing the time duration, minimizing the fuel consumption, maximizing the lading cross range, etc. However, it is worth noting that it is only in the recent five years that there has been a growing interest in planning the flight trajectories with the consideration of multiple mission objectives. In a large number of practical trajectory design problems, multiple performance measures should be frequently considered during the decision making phase and this brings the development of multi-objective trajectory optimization (MOTO)[71-73].

Similar with the definition given by Eq.(5), a standard multi-objective op- 
timization problem can be written as:

$$
\begin{array}{ll}
\text { Find decision variables } & x=\left[x_{1}, x_{2}, \ldots, x_{n}\right] \\
\text { Minimize objective function } & J(x)=\left[J_{1}(x), J_{2}(x), \ldots, J_{M}(x)\right] \\
\text { subject to } & x_{\min } \leq x \leq x_{\max } \\
& H_{i}(x)=0 \\
& G_{j}(x) \leq 0 \\
& (i=1,2, \ldots, E) \\
& (j=1,2, \ldots, I)
\end{array}
$$

where $M$ stands for the number of mission objectives considered in the problem.

The main objective of this section is to report the latest development of multi-objective optimization strategies for producing the optimal trajectories of different spacecraft flight missions. Moreover, the key features such as the advantages and disadvantages of using these recently-developed multi-objective optimization techniques are also discussed. Based on these reported results, readers may gain a more clear understanding with respect to the performance and behaviours of different multi-objective optimization algorithms for addressing various space vehicle flight missions. Moreover, it is possible for the readers to formulate their own algorithm or improve one of these existing techniques in order to address their specific space vehicle trajectory design problems.

We classify different MOTO methods existing in the literature into two groups. That is, the multi-objective evolutionary algorithm-based techniques and the multi-objective transcription method-based strategy. The most popular methods among these two groups are summarised and reported in Table 3 and Table 4. It is important to highlight that not all the MOTO methods under each category are listed in this table. Alternatively, only some important instances are reviewed and these techniques are discussed in detail in the following subsections.

\subsection{Multi-Objective Evolutionary Algorithms}

There exist many multi-objective algorithms which can be applied to solve the MOTO problem [91, 92]. One way to solve the MOTO problem is to use the principle of "pareto-optimal" [93-95]. A pareto-optimal solution is optimal in the sense that no other solutions are superior (better) to it in the current searching space when all objectives are considered [96, 97]. Since it is usually 
Table 3: Popular multi-objective evolutionary algorithms available for trajectory design problems

Multi-objective evolutionary algorithm-based techniques

Nondominated sorting genetic algorithm II (NSGA-II) [74]

Improved nondominated sorting genetic algorithm II (I-NSGA-II) [75]

Nondominated sorting genetic algorithm III (NSGA-III) [76]

Multi-objective evolutionary algorithm Based on decomposition (MOEA/D) [77]

Multi-objective particle swarm optimization (MOPSO) [78]

Multi-objective adaptive particle swarm optimization (MOAPSO) [79]

Multi-objective adaptive gradient particle swarm optimization (MOAGPSO) [80]

Multi-objective artificial bee colony (MOABC) [81]

Niched pareto genetic algorithm (NPGA) [82]

Strength pareto particle swarm optimization (SPPSO) [83]

Adaptive differential evolution and modified game theory (ADEMGT) [84]

Table 4: Popular multi-objective transcription methods available for trajectory design problems

\begin{tabular}{l}
\hline \hline Multi-objective transcription-based techniques \\
\hline Weighted-sum method (WS) [85] \\
Physical programming method (PP) [86] \\
Fuzzy physical programming (FPP) [33] \\
Interactive physical programming (IPP) [87] \\
Interactive fuzzy physical programming (IFPP) [88] \\
Goal programming (GP) [89] \\
Fuzzy goal programming (FGP) [75] \\
Fuzzy satisfactory goal programming (FSGP) [32] \\
Adaptive surrogate model (ASM) [90]
\end{tabular}

hard to find a solution that can optimize all the objectives, it is then interesting to find all the pareto-optimal solutions and create the pareto-optimal set.

To find the pareto-optimal set, the evolutionary multi-objective optimization (EMO) methodology has been analyzed as a promising method to visualize the relationships between objectives and calculate the pareto-front [77]. The general steps of using EMOs to solve MOTO problems can be summarised as follows: 
1. Initialize the population and other control parameters of the EMO algorithm.

500

505

2. For each candidate among the population/swarm, calculate the objective function values $J$ and the constraint violation value.

3. Generate offspring population/swarm by using various evolutionary strategy.

4. Combine the offspring population/swarm with the previous population.

5. Calculate the fitness value for each candidate using the information of objective function values and constraint violation value.

6. Assign all non-dominated ranks using the pareto dominant rule.

7. According to the selection operator, select the best set of individuals as the candidates of the new generation.

8. Repeat step 2-7 until the maximum iteration number is reached.

New EMO techniques and applications have been widely applied in aerospace engineering during the past decades [98, 99]. For example, in terms of the theoretical development, the authors in [63] proposed an optimal path control strategy for addressing general multi-objective optimization problems. Ji et al. [100] designed a modified NSGA-II algorithm to address a multi-objective allocation problem. In [101], the authors proposed a decomposition-based sorting technique for handling benchmark multi-objective problems.

Regarding the practical applications, a constrained multi-objective evolutionary algorithm (MOEA) was applied in [13] in order to solve a bi-objective reentry trajectory design problem. The authors in [102] considered a low-thrust gravity assist trajectory design problem. In their work, two contradicting mission objectives, minimizing the flight time duration and minimizing the fuel consumption, were considered and the pareto front was successfully produced by applying the NSGA-II algorithm. Similarly, an enhanced NSGA-II algorithm was proposed in [103], wherein a specific migration scheme was embedded in the original algorithm framework. This improved method was then applied to solve an Earth-Jupiter orbital transfer problem with the consideration of different fly-by sequences.

In [4], the authors extended the standard PSO algorithm to a multi-objective version, thereby constructing a multi-objective PSO (MOPSO) algorithms. This extended algorithm was then applied to address a Earth-Jupiter-Saturn orbital 
transfer problem and the results illustrated the feasibility as well as the reliability of the proposed method.

In [76], the authors proposed an extended NSGA-III algorithm in order to 535 address the multi-objective spacecraft reentry trajectory design problem with the consideration of path constraints and no-fly zone constraints. This method applied a set of reference points so as to guide the evolutionary direction. By applying the proposed method, the contradicting relationships between different mission objectives can be reflected successfully. Moreover, it was found that by

${ }_{540}$ applying the reference point strategy, the obtained pareto front solution can be more well-distributed and optimal.

Although the contradicting relationship between objectives could be reflected and the pareto set was obtained, the computational burden due to the optimization process is high. In addition, a main challenge faced by MOEAs is

${ }_{545}$ that it has the restriction of dimensionality in solving problems containing more than three objectives. This is because the current domination principle which is usually used and embedded in the MOEA framework lacks the ability to provide an adequate selection pressure and emphasize feasible solutions [74, 77]. In other words, the selection pressure can hardly be allocated to each objective uniformly, thereby resulting in poor diverse representation of the pareto front.

\subsection{Multi-Objective Transcription Methods}

Currently, most of the existing studies are focusing on the development or implementation of MOEAs for general MOPs [77, 99, 104-106]. This type of technique is effective for analyzing the relations between objectives and generate the pareto front. However, since all the objectives are involved in the optimization iteration and rank sorting process, the computational complexity can be high. Moreover, if the different types of preference requirements are required to be taken into account, the MOEA-based approach might need to rely on the interactive process, which is still a challenging problem for the decision makers.

Due to these drawbacks and challenges, in this subsection, the multi-objective transcription (MOT) strategy is outlined. This type of algorithm aims to solve 
the multi-objective optimization problem in the form of

$$
\begin{array}{ll}
\text { Find decision variables } & x=\left[x_{1}, x_{2}, \ldots, x_{n}\right] \\
\text { Minimize objective function } & J(x)=\left[J_{1}(x), J_{2}(x), \ldots, J_{M}(x)\right] \\
\text { subject to } & x_{\min } \leq x \leq x_{\max } \\
& H_{i}(x)=0 \\
& G_{j}(x) \leq 0 \\
& J(x) \in \mathscr{P} \\
& (i=1,2, \ldots, E) \\
& (j=1,2, \ldots, I)
\end{array}
$$

in which $\mathscr{P}=\left\{J(x) \mid P\left(J_{i}(x)\right)>P\left(J_{j}(x)\right)\right\}$. Here $P(\cdot)$ denotes priority factors of different performance indices. The inequality $P\left(J_{i}(x)\right)>P\left(J_{j}(x)\right)$ can be understood as regarding the priority of the $i$ th objective higher than the $j$ th objective. It is desired to find a proper algorithm that is effective to handle the mission-dependent preference constraints and reduce the computational complexity. The general idea of the MOT strategies is to reformulate the original multi-objective formulation to a single-objective problem (SOP). Compared with MOEA strategies studied in the previous subsection, the MOT methods have the capability to handle the preference requirement (e.g. the priority constraints) and does not rely on the time-consuming rank sorting process.

A typical MOT example that has been widely used in the literature is the weighted-sum method where weight coefficients are used to transform different criterions into only one single objective. However, it was investigated in [88] that the weight coefficients may fail to represent the true preferences or priorities. Gao et al. [11] computed the optimal control command with respect to a multiobjective spacecraft rendezvous task. In their work, the multi-objective optimal control problem was transcribed into a convex optimization issue subject to linear matrix inequality constraints. However, this formulation can hardly be extended to solve the multi-objective optimal control problems with simultaneous consideration of priority requirements.

In 1996, Messac designed a physical programming (PP) approach to convert the objectives [86], which removes the information of priority and weight coefficients. This method divided the objective value into different preference regions as indicated in Fig.3. Subsequently, a physical optimization model was established to represent the original problem formulation.

In their follow-up research [87], an interactive strategy was proposed and 


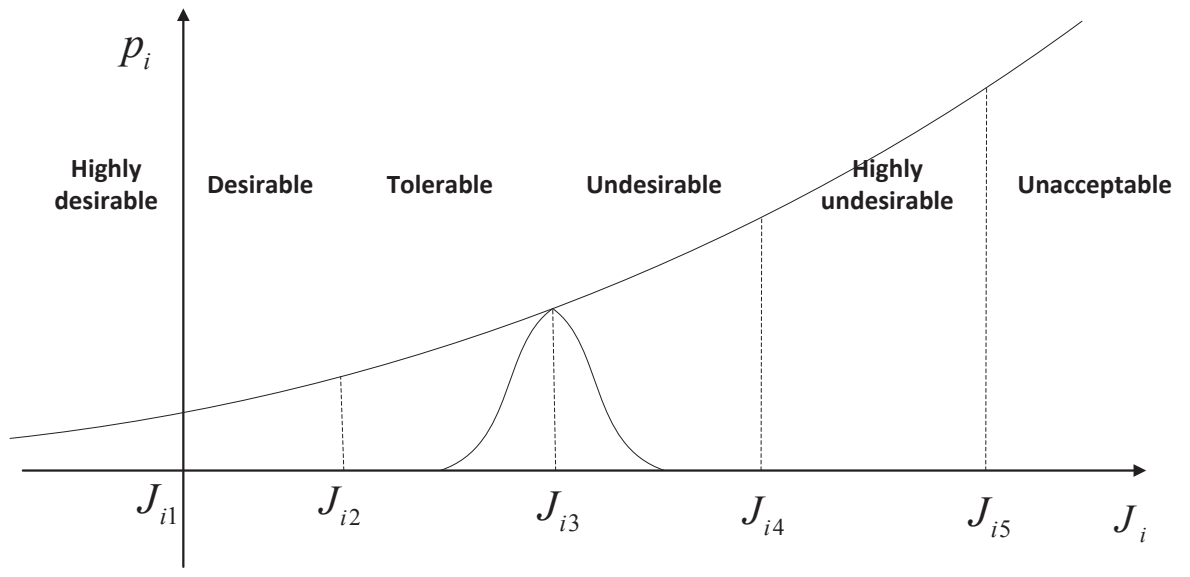

Figure 3: Preference region illustration

embedded in the PP framework, thereby constructing an interaction physical programming scheme (IPP). However, since the formulated optimization model is strongly nonlinear, selecting the preference function and formulating the preference model are still difficult.

To solve this problem, in [33], the authors proposed an enhanced PP method by applying the definition of fuzzy set. This enhanced technique was then applied to address a multi-objective space maneuver vehicle trajectory design problem and the results confirmed its feasibility. Besides, in [88], an interactive process was designed and embedded in the algorithm framework as illustrated in Fig.4. The interactive process is achieved by adjusting the aspiration level and preference functions, thus allowing more control flexibility to the decision maker.

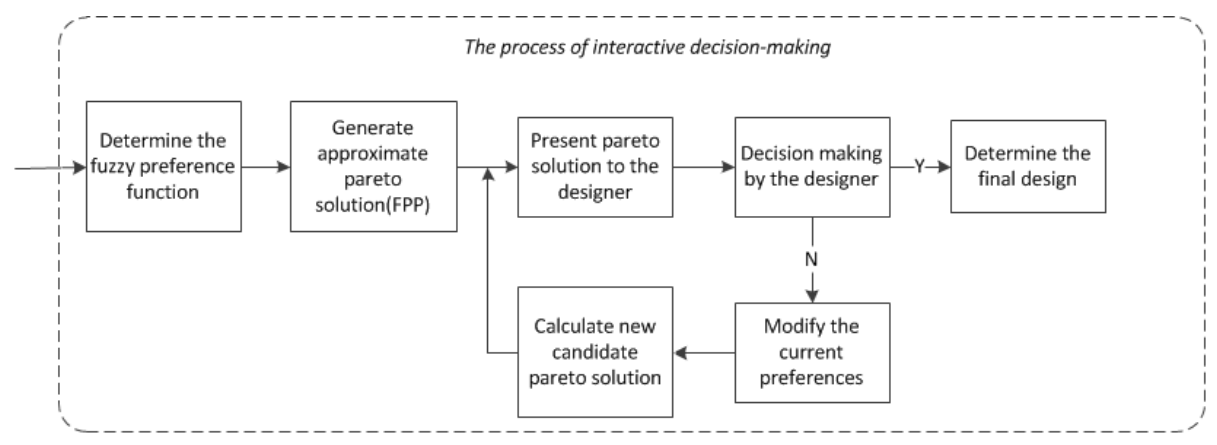

Figure 4: Graphical illustration of the interactive decision-making process 


\section{Stochastic Spacecraft Trajectory Optimization}

Although most previously reported optimization-based techniques have been shown to be effective and reliable tools for generating optimal flight trajectories at deterministic models. It should be noted that in many real-world mission scenarios, various model or actuator uncertainties must frequently be taken into 
account during the trajectory planning phase. As a result, a proper treatment of the dynamics and constraints affected by stochastic variables is requested, which [107-114].

This section investigates various computational frameworks existing in the literature for addressing the problem of space vehicle trajectory optimization with the consideration of chance constraints (CCs) and stochastic dynamics. craft trajectory optimization with deterministic dynamic model. The strategies that are available to deal with stochastic variables involved in the constraints will be discussed in detail. Following that, in Section 7.2, the problem of chanceconstrained spacecraft trajectory optimization with stochastic dynamics will be outlined. We hope that by reading this section, readers can gain a better understanding in terms of the definitions, solution approaches, and current challenges of the stochastic spacecraft trajectory design problems.

\subsection{Chance-Constrained Spacecraft Trajectory Optimization}

In this subsection, we firstly consider the chance-constrained spacecraft trajectory optimization problem with deterministic dynamics or equations of motion. That is, only the flight path constraints are affected by some uncertain variables. The formulation of this type of problem can be obtained by introducing the uncertain variable and probabilistic constraints in Eq.(4), which has the following form:

$$
\begin{array}{clr}
\text { minimize } & J=\Phi\left(x_{0}, t_{0}, x_{f}, t_{f}\right)+\int_{t_{0}}^{t_{f}} L(x(t), u(t), t) d t \\
\text { subject to } & \forall t \in\left[t_{0}, t_{f}\right] & \\
& \dot{x}(t)=f(x(t), u(t), t) & \text { (dynamic constraints) } \\
& b_{L} \leq b\left(x_{0}, t_{0}, x_{f}, t_{f}\right) \leq b_{U} & \text { (boundary conditions) } \\
& \operatorname{Pr}\left\{b_{L} \leq b\left(x_{0}, t_{0}, x_{f}, t_{f}\right) \leq b_{U}\right\} \geq 1-\epsilon_{\phi} \quad \text { (terminal CCs) } \\
& \operatorname{Pr}\left\{g_{L} \leq g(x(t), u(t), t ; \xi) \leq g_{U}\right\} \geq 1-\epsilon_{g} \quad \text { (path CCs) }
\end{array}
$$

In Eq.(8), without loss of generality, it is supposed that $\xi \in \Omega \subset \mathbb{R}^{N_{p}}$ is an uncertain parameter with a known probability density function (PDF). The noise-perturbed path function is defined by $g: \mathbb{R}^{N_{x}} \times \mathbb{R}^{N_{u}} \times \mathbb{R} \times \Omega \mapsto \mathbb{R}^{N_{g}}$. $\operatorname{Pr}(\cdot)$ stands for the probability operation, whereas $\epsilon$ stands for the acceptable probability of occurrence. It should be noted that the path chance constraint 
in Eq.(8) is a joint chance constraint. It reveals the fact that in order for the joint event $\left\{g_{L} \leq g(x(t), u(t), t ; \xi) \leq g_{U}\right\}$ to be true, each individual component of the vector function $g$ should probabilistically satisfy $g_{L}^{i} \leq g^{i} \leq g_{U}^{i}, i=$ $1,2, \ldots, N_{g}$. A common way to handle the joint chance constraint is to decompose it into individual scalar chance constraints. This strategy might be conservative yet computationally more attractive. Specifically, using Boole's inequality, a sufficient condition of the original joint chance constraint can be obtained [108]:

$$
\operatorname{Pr}\left\{g^{i}(\xi)<g_{L}^{i}\right\} \leq \epsilon_{1, i}, \quad \operatorname{Pr}\left\{g^{i}(\xi)>g_{U}^{i}\right\} \leq \epsilon_{2, i}
$$

655 Then, by imposing $\sum_{i=1}^{N_{g}}\left(\epsilon_{1, i}+\epsilon_{2, i}\right)<\epsilon_{g}$, the joint chance constraint can be satisfied.

Compared with designing a completely new solution approach, it is more interesting to apply standard optimal control solvers to optimize the state and control trajectories. However, the formulation shown in Eq.(8) cannot be solved in its present form. This is because the evaluation of Eq.(11) can hardly be performed. Therefore, a proper treatment of the constraint influenced by stochastic parameters is required, and solving the chance-constrained optimization problem reduces to tackling the non-deterministic chance constraints. A commonly used strategy is to transcribe the probabilistic constraint (11) into a deterministic one, and this brings the development of robust trajectory planning [107] and chance-constrained optimal path design [108].

Robust trajectory planning is based on robust optimization (RO) algorithms. The main advantage with the RO method is that it is easy to apply and simple to understand. In recent years, a large amount of research work has been reported in this field [112, 115-118]. In particular, Li and Shi [116] designed a robust distributed model predictive control scheme for a class of nonlinear multiagent system. In their work, the model uncertainty was handled by introducing a robustness constraint in the optimization model. In [117], authors proposed a differential evolution-based technique to solve the minimax optimization problems that naturally arise in practical robust designs. Wang and Pedrycz [118] developed an adaptive data-driven RO method in order to solve a class of optimization problem with the consideration of parameter uncertainty. Moreover, a new robust optimization methodology, named active robust optimization, was investigated in [112]. It is well known that the RO formulation aims to find the solution of the worst-case optimization scenario. This indicates that the 
calculated solution can satisfy all constraints with respect to any realization of the stochastic parameters. In other words, constraint violations are not allowed in an RO formulation.

Alternatively, chance-constrained optimal path design relies on chanceconstrained optimization (CCO) algorithms. This type of algorithm allows constraint violations to be less than a user-specified risk parameter. A detailed review regarding different $\mathrm{CCO}$ algorithms can be found in [119] and the references therein. In [120], the authors proposed a CCO-based model predictive control scheme so as to optimize the movement of the ego vehicle. Considering the uncertainty in the system state as well as the constraint, a hybrid CCO method was designed in [121] and applied to solve an autonomous vehicle motion planning problem. Though applying RO methods can achieve the strongest solution feasibility, the $\mathrm{CCO}$ methods tend to be less conservative.

However, one challenge of the use of CCO methods is that the probabilistic functions and their derivatives cannot be calculated directly. An effective strategy to handle this issue is to replace or approximate these constraints by using deterministic functions or samples [122-124]. The motivation for the use of approximation-based strategies relies on their ability in dealing with general probability distributions for the uncertainty as well as preserving feasibility of approximation solutions. Until now, some approximation techniques have been proposed based on the Bernstein method [108, 122], the constraint tightening approach [125], the scenario approximation [126], etc. The chance-constrained optimal path design reported in these works usually employed a discretization technique to parameterize uncertain variables and create the trajectory ensemble. Subsequently, the resulting discretized version of the problem was solved by applying standard optimal control solvers. In order to provide reliable gradient information to the optimization algorithm, different chance constraint approximation methods were proposed to replace the original probability constraints. The established methodology was then implemented to explore the optimal trajectories for different spacecraft flight trajectory planning scenarios with the consideration of probabilistic constraints. Simulation results and comparative studies demonstrated that these proposed chance constraint handling strategies can outperform other existing robust optimization-based approaches, and these computational frameworks can produce reliable and less conservative solutions for different chance-constrained spacecraft trajectory planning problems. 
Based on the discussion stated above, a popular solution-finding procedure for addressing the chance-constrained spacecraft trajectory design problem can be summarised to the following four steps:

1. Decompose the joint chance constraint.

2. Approximate the chance constraints.

3. Transcribe the original problem formulation into a deterministic version.

4. Solve the problem using standard trajectory optimization solver.

Although the aforementioned CCO-based strategies can be feasible for replacing the probabilistic constraints, there are still some open problems. For example, an important issue is that the conservatism is usually high and difficult to be controlled. Furthermore, the smoothness, differentiability and convergence properties of the approximation strategy can hardly be preserved.

\subsection{Chance-Constrained Spacecraft Trajectory Optimization: Stochastic Dy- namics}

This subsection firstly outlines the problem of chance-constrained spacecraft trajectory optimization with noise-perturbed system dynamics. Its formulation can be easily obtained by extending Eq.(8) with the introduction of uncertain variable in the dynamics. Specifically, it can be written in the form of:

$$
\begin{array}{clc}
\text { minimize } & J=J=\mathbb{E}\left[\Phi\left(x_{0}, t_{0}, x_{f}, t_{f}\right)+\int_{t_{0}}^{t_{f}} L(x(t), u(t), t) d t\right] \\
\text { subject to } & \forall t \in\left[t_{0}, t_{f}\right] \\
& \dot{x}(t)=f(x(t), u(t), t)+\xi \quad \text { (noise-perturbed dynamics) } \\
& b_{L} \leq b\left(x_{0}, t_{0}, x_{f}, t_{f}\right) \leq b_{U} \quad \text { (boundary conditions) } \\
& \operatorname{Pr}\left\{b_{L} \leq b\left(x_{0}, t_{0}, x_{f}, t_{f}\right) \leq b_{U}\right\} \geq 1-\epsilon_{\phi} \quad \text { (terminal CCs) } \\
& \operatorname{Pr}\left\{g_{L} \leq g(x(t), u(t), t) \leq g_{U}\right\} \geq 1-\epsilon_{g} \quad \text { (path CCs) }
\end{array}
$$

Due to the nature of the stochastic dynamics and constraints, the performance index $J$ is formulated in an expectation form. The term $\Phi$ denotes the expected Mayer cost and the term $L$ is the expected process cost. Compared with the problem formulation given by Eq.(8), an important change of Eq.(9) is that the system dynamics are noise-perturbed. It should be noted that this noise-perturbed system model has a rather simple type of motion which can be treated as conditionally deterministic. This is because if the uncertain parameter $\xi$ is known, then the system motion would be known at all future time 
instants, which indicates that the system motion is deterministically conditioned on the knowledge of the uncertain parameter $\xi$. Although conditionally deterministic motion tends to be more restrictive than the stochastic motion, it is still worthy to be deeply researched as there are many real-world mission scenarios where the system equations are explicitly related to some uncertain parameters $[127]$.

Different from Eq.(9), a more general form of the stochastic spacecraft trajectory optimization model can be defined by considering the uncertain effect as a stochastic process. This indicates that the system dynamics is constructed as a set of stochastic differential equations (SDEs). Then the overall problem formulation is described in Eq.(10) [128]:

$$
\begin{aligned}
\text { minimize } & J=J=\mathbb{E}\left[\Phi\left(x_{0}, t_{0}, x_{f}, t_{f}\right)+\int_{t_{0}}^{t_{f}} L(x(t), u(t), t) d t\right] \\
\text { subject to } \quad \forall t \in\left[t_{0}, t_{f}\right] & d x(t)=f(x(t), u(t), t) d t+G(x(t), u(t), t) d \xi(t) \quad \text { (SDEs) } \\
& b_{L} \leq b\left(x_{0}, t_{0}, x_{f}, t_{f}\right) \leq b_{U} \quad \text { (boundary conditions) } \\
& \operatorname{Pr}\left\{b_{L} \leq b\left(x_{0}, t_{0}, x_{f}, t_{f}\right) \leq b_{U}\right\} \geq 1-\epsilon_{\phi} \quad \text { (terminal CCs) } \\
& \operatorname{Pr}\left\{g_{L} \leq g(x(t), u(t), t) \leq g_{U}\right\} \geq 1-\epsilon_{g} \quad \text { (path CCs) }
\end{aligned}
$$

where $\xi(t)$ stands for the stochastic process, while $f$ and $G$ reflect the drift and diffusion parts of the random state process. In the problem formulation (10), the uncertainty is now considered as a dynamic variable. Therefore, the solution to this problem is no longer a deterministic control sequence. Although some works can be found in the literature to explore this problem, they all target at a simplified linear quadratic version [128]. Practical numerical algorithms for the solution to a general stochastic spacecraft trajectory optimization problem are still at an initial development stage.

To solve the problem (9), an important procedure is to apprximate/discretize the uncertain variables appearing inside the dynamics. To achieve this, a certain stochastic quadrature formula (SQF) should be used to achieve the approximation with a desired error order (raft of convergence). An SQF of degree $N$ can be regarded as a set of weighted parameters $\left\{w_{k}\right\}, k \in\{1,2, \ldots N\}$ and $N_{p^{-}}$ dimensional uncertain variables $\left\{\xi_{k}\right\}, k \in\{1,2, \ldots N\}$ such that the equation $\sum_{k=1}^{N} w_{k} F\left(\xi_{k}\right) \approx \int F(\xi) R(\xi) d \xi$ holds true. Here, $R(\xi)$ is the PDF of $\xi$. With the introduction of SQF, an approximation of the stochastic integral can then 
be built such that

$$
\mathbb{E}[F(x, u, \xi)] \approx \sum_{k=1}^{N} w_{k} F\left(x, u, \xi_{k}\right)
$$

where $\mathbb{E}(\cdot)$ denotes the expectation operator.

Until now, there are many effective SQF methods and their variances that have been reported for quantifying the uncertain variables. Among them, generalized polynomial chaos (gPC) theory-based approaches have attracted great attention due to their ability in decomposing the stochastic variables into a convergent series of polynomials. gPC methods have been widely applied in various aerospace engineering applications [107, 129]. The general procedure of this type of strategy is to utilize deterministic orthogonal polynomials and coefficients for deriving the expression of stochastic systems. It was shown in [129] that the gPC-based techniques can be efficient for optimal control problems containing a relatively small number of stochastic variables. For example, if the dynamic equations defined in (9) contain a relatively small number of stochastic variables, the gPC-based algorithms can be used to interpret the uncertain effects. Most of the chance-constraint handling strategies can still be applied to handle the probabilistic constraints existing in Eq.(9). However, for the uncertain trajectory optimization problem with a relatively large number of uncertain parameters, this type of method becomes computationally expensive and they are no longer suitable for representing the uncertainty for the problem (9).

Another well-developed class of SQF techniques is the sampling-based methods. Typical examples include the Markov chain Monto Carlo (MCMC) approach and quasi-Monto Carlo methods. The motivation for the use of samplingbased methods relies on their simplicity and the fact that the approximation error order is independent with respect to the dimension of $\xi$. Take MCMC as an example, a stochastic variable ensemble $\{\xi\}_{k=1}^{N}$ can be constructed by randomly sampling from the probability distribution (e.g. $\{\xi\}_{k=1}^{N} \sim R(\xi)$ ). Each sample will be weighted equally (e.g. $\left.w_{k}=N^{-1}\right)$, thereby producing an $\mathcal{O}(1 / \sqrt{N})$ convergence rate in terms of the approximation error. Therefore, for most published works, the MCMC technique was employed to model the uncertain parameters. However, in order to deal with the noise-perturbed dynamics, a propagation process might need to be performed. This process is not easy to be executed due to the nonlinearity of the system model. Moreover, as the dynamics are 
propagated, all the system states are required to satisfy the probabilistic constraints as shown in Eq.(9). This will inevitably increase the computational complexity and computational time [108]. In addition, the accumulation of constraint violations and conservatism might damage the optimality of the solution as well as the convergence ability of the optimization algorithm.

Therefore, it is obvious that more efforts should be made in order to tackle the issues mentioned previously. And there is still a lot of room for improvement with respect to applying or designing new strategies in chance-constrained spacecraft trajectory optimization problems under the consideration of noiseperturbed and stochastic dynamics.

\section{Recent Practical Applications of the optimized trajectory}

In recent years, a number of new practical application fields have been expanded due to the development of trajectory optimization techniques. The focus of this section is on the recent applications of the optimal flight trajectories from a high-level perspective. Various application scenarios for which the optimal trajectories have been successfully implemented or are under strong scientific investigation are surveyed. These scenarios include: the design of in-

tegrated spacecraft guidance and control systems, the design of spacecraft or satellites formation control schemes, and the design of a database-based online guidance strategy.

\subsection{Design of Integrated Spacecraft Guidance and Control Systems}

One important functionality of the optimal flight trajectory is that it can contribute to the design of spacecraft online guidance and control systems [130-132]. Works on developing this topic can be easily found in the recentlypublished articles. In [133], a segmented predictor-corrector guidance approach was designed for the Mars entry capsule. In this work, an optimal flight path was pre-planned and several way-points were selected as the segmented targets. so Subsequently, the traditional predictor-corrector approach was applied for these segments. It was shown in the simulation that this strategy can reduce the computational time and preserve the advantage of the standard predictor-corrector approach. Similarly, the work of Dai and Xia [134] implemented an optimal Mars entry trajectory so as to design a terminal sliding mode control-based guidance law for the Mars landing problem. In their work, the terminal sliding 
mode control scheme was designed to remove the deviation between the actual flight trajectory and the pre-designed optimal reference trajectory. Moreover, an extended state observer was used to measure the uncertain term in the vehicle dynamics.

Traditionally, spacecraft guidance and control systems are designed separately as two loops [135]. That is, an inner loop autopilot is established so as to track the angular command generated by the outer-loop guidance scheme. However, such a two-loop design usually leads to large design iterations and does not fully exploit the relationships between different subsystems, thereby resulting ${ }_{845}$ in a suboptimal performance [136]. In recent years, there has been a growing interest in the design of integrated guidance law and flight control systems. For instance, Tian et al. [8] and Liu et al. [137] proposed the integrated trajectory and attitude coordination control schemes in order to control different types of spacecraft in near real-time. Basically, there are three main parts in their designed system: an offline trajectory optimization component, an online optimal feedback guidance component, and a spacecraft attitude controller. In the offline trajectory optimization component, a reference flight trajectory is firstly generated via well-developed trajectory optimization strategies reviewed in the previous sections. Then, the reference is provided to the online optimal feedback guidance component, where a reference-tracking algorithm is used to produce the control increment which will be applied as the reference control commands for the inner attitude control system. The core aim for this integrated design is to steer the vehicle such that it can fly along the pre-specified reference path.

\subsection{Design of Spacecraft/Satellite Formation Control Schemes}

The formation control of spacecraft/satellites aims to steer a fleet of smallscale spacecrafts/satellites to follow a predefined trajectory while preserving a desired pattern. It is one of the most popular directions that can contribute to the future development of space technology and exploration of the space [138-140]. There are many effective formation control methods existing in the literature. Among them, the leader-follower method has attracted great attention due to its ability in dealing with complex tasks. This type of method can be referred to the fact that some spacecraft among the fleet serve as the leader, whereas the remaining spacecraft will act as the followers. The general idea is to force the followers to track the trajectory of the leader in order to keep the formation and fulfill other mission requirements. Therefore, a well-designed flight 
trajectory, particularly for the leading vehicle, is a key for stable flight and for improved control of the system. In the recent year, a large amount of research has been carried out by incorporating trajectory optimization techniques and attitude tracking control methods for this kind of problem. For example, in [141], the authors combined trajectory optimization and configuration control to study the problem of orbital maneuver for a formation system. In addition, considering the communication distance and the ground projection area as constraints, a constrained trajectory optimization method, together with a coordinative control strategy was proposed and successfully applied to a micro space vehicle formation flying problem in [142]. Furthermore, a deep-space dual-spacecraft formation flying problem was studied in [143], wherein the optimal obstacle avoidance flying trajectory was produced via a standard trajectory optimization method.

\subsection{Database-based Online Guidance Strategy}

Recently, a growing interest can be found in developing the online guidance strategy based on a large pre-planned optimal trajectory set. Contributions to this kind of approach are reported in the literature [144, 145]. For example, in [145] a database-based online guidance scheme was designed and applied for the space vehicles reentry problem. In this guidance scheme, a large database of optimal trajectories was firstly generated. Following that, the optimal command solution corresponding to a subset of initial-conditions variations was produced by the onboard algorithm. The main advantage of using such a design is that the real-time applicability can be easily achieved and compared with other guidance schemes based on the control theory, it is more reliable to be applied online. In addition, this type of method is able to deal with dispersions during the flying phase. Therefore, we believe that more success stories in applying the database-based online guidance strategies are going to appear in the next couple of decades.

\section{Conclusions and Future Development}

The trajectory design of spacecraft is usually recognized as an optimal control problem and the selection of optimization method has a decisive influence on the final flight trajectory. A survey of newly-developed optimization strategies that are available for addressing constrained spacecraft trajectory design 
problems has been given in this investigation. Various spacecraft trajectory 905 optimization approaches and achievements have been reviewed in this paper. It is worth noting that the progresses in the field of nonlinear programming as well as artificial intelligent-based optimization have recently resulted in a large amount of numerical trajectory design approaches. Applying these new methods can not only fulfill complex mission requirements but also improve the quality of the obtained solution even when multiple constraints are required to be considered.

Due to the fact that in practice, more than one mission objective and different types of vehicular or environmental uncertainties may exist in the path planning phase, the original problem formulation should be extended to a multiobjective trajectory planning version or a stochastic trajectory planning version. In this article, we have reviewed the up-to-date theoretical development in terms of the optimization of the space vehicle flight trajectory with emphasis on multiple conflicting mission performance indices and stochastic variable/constraints and handling strategies. Key features such as the advantages and disadvantages of using these recently-developed techniques were described, and guidelines were given with respect to the development of reliable multi-objective and stochastic spacecraft trajectory optimization algorithms. Some recent applications of the optimized trajectory were also discussed to clearly show that a widespread implementation of these techniques and results has already begun.

We believe that many more success stories in applying the multi-objective trajectory optimization as well as the stochastic trajectory optimization techniques in various domains such as interplanetary travel, rendezvous and docking, formation flying, and planetary exploration will be reported in the next few decades. For many of these new spacecraft tasks, novel multi-objective and stochastic trajectory optimization algorithms are urgently needed for handling different mission-dependent or vehicle-dependent performance indices and model errors/uncertainties. Moreover, works on the validation of the effectiveness, reliability and optimality of the trajectory optimization methods are highly likely to appear. This will give the aerospace engineers a clear view of 935 the algorithm credibility. 


\section{References}

[1] J. T. Betts, Survey of numerical methods for trajectory optimization, Journal of Guidance, Control, and Dynamics 21 (2) (1998) 193-207. doi: $10.2514 / 2.4231$.

[2] B. A. Conway, A survey of methods available for the numerical optimization of continuous dynamic systems, Journal of Optimization Theory and Applications 152 (2) (2012) 271-306. doi:10.1007/s10957-011-9918-z.

[3] J. Lavaei, A. Momeni, A. G. Aghdam, A model predictive decentralized control scheme with reduced communication requirement for spacecraft formation, IEEE Transactions on Control Systems Technology 16 (2) (2008) 268-278. doi:10.1109/TCST .2007.903389.

[4] F. Alonso Zotes, M. Santos Penas, Particle swarm optimisation of interplanetary trajectories from earth to jupiter and saturn, Engineering Applications of Artificial Intelligence 25 (1) (2012) 189-199. doi:https: //doi.org/10.1016/j. engappai.2011.09.005.

[5] R. Chai, A. Savvaris, A. Tsourdos, S. Chai, Y. Xia, Optimal fuel consumption finite-thrust orbital hopping of aeroassisted spacecraft, Aerospace Science and Technology 75 (2018) 172-182. doi:https://doi.org/10. $1016 / j$.ast. 2017.12 .026 .

[6] A. Bogorad, C. Bowman, A. Dennis, J. Beck, D. Lang, R. Herschitz, M. Buehler, B. Blaes, D. Martin, Integrated environmental monitoring system for spacecraft, IEEE Transactions on Nuclear Science 42 (6) (1995) 20512057. doi:10.1109/23.489252.

[7] R. Chai, A. Savvaris, A. Tsourdos, S. Chai, Y. Xia, Optimal tracking guidance for aeroassisted spacecraft reconnaissance mission based on receding horizon control, IEEE Transactions on Aerospace and Electronic Systems 54 (4) (2018) 1575-1588.

[8] B. Tian, W. Fan, R. Su, Q. Zong, Real-time trajectory and attitude coordination control for reusable launch vehicle in reentry phase, IEEE Transactions on Industrial Electronics 62 (3) (2015) 1639-1650. doi: 10.1109/TIE. 2014.2341553. 
[9] K. F. Graham, A. V. Rao, Minimum-time trajectory optimization of lowthrust earth-orbit transfers with eclipsing, Journal of Spacecraft and Rockets 53 (2) (2016) 289-303. doi:10.2514/1.A33416.

[10] A. V. Rao, S. Tang, W. P. Hallman, Numerical optimization study of multiple-pass aeroassisted orbital transfer, Optimal Control Applications and Methods 23 (4) (2002) 215-238. doi:10.1002/oca.711.

[11] H. Gao, X. Yang, P. Shi, Multi-objective robust h-infinity control of spacecraft rendezvous, IEEE Transactions on Control Systems Technology 17 (4) (2009) 794-802. doi:10.1109/TCST .2008.2012166.

[12] M. Pontani, B. A. Conway, Optimal finite-thrust rendezvous trajectories found via particle swarm algorithm, Journal of Spacecraft and Rockets 50 (6) (2013) 1222-1234. doi:10.2514/1.A32402.

[13] C. Gan, W. Zi-ming, X. Min, C. Si-lu, Genetic Algorithm Optimization of RLV Reentry Trajectory, International Space Planes and Hypersonic Systems and Technologies Conferences, American Institute of Aeronautics and Astronautics. doi:doi:10.2514/6.2005-3269.

[14] Z. Kenan, C. Wanchun, Reentry Vehicle Constrained Trajectory Optimization, International Space Planes and Hypersonic Systems and Technologies Conferences, American Institute of Aeronautics and Astronautics, 2011. doi:doi:10.2514/6.2011-2231.

[15] A. Rajesh, Reentry Trajectory Optimization: Evolutionary Approach, Multidisciplinary Analysis Optimization Conferences, American Institute of Aeronautics and Astronautics, 2002. doi:doi:10.2514/6.2002-5466.

[16] W. Robert, A. Mark, B. Jeffrey, W. Robert, A. Mark, B. Jeffrey, Minimum heating reentry trajectories for advanced hypersonic launch vehicles, Guidance, Navigation, and Control and Co-located Conferences, American Institute of Aeronautics and Astronautics, 1997. doi:doi: $10.2514 / 6.1997-3535$.

[17] I. Mikhail, V. Pavel, K. Alexandr, Numerical Investigation of the EXPERT Reentry Vehicle Aerothermodynamics Along the Descent Trajectory, Fluid Dynamics and Co-located Conferences, American Institute of Aeronautics and Astronautics, 2007. doi:doi:10.2514/6.2007-4145. 
[18] I. M. Ross, M. Karpenko, A review of pseudospectral optimal control:

[20] A. V. Rao, A survey of numerical methods for optimal control, Advances in the Astronautical Sciences 135 (1).

[21] J. T. Betts, W. P. Huffman, Mesh refinement in direct transcription methods for optimal control, Optimal Control Applications and Methods 19 (1)

[22] H. Yang, H. Baoyin, Fuel-optimal control for soft landing on an irregular asteroid, IEEE Transactions on Aerospace and Electronic Systems 51 (3) (2015) 1688-1697. doi:10.1109/TAES.2015.140295.

[23] H. Yang, X. Bai, H. Baoyin, Rapid generation of time-optimal trajectories for asteroid landing via convex optimization, Journal of Guidance, Control, and Dynamics 40 (3) (2017) 628-641. doi:10.2514/1.G002170.

[24] I. M. Ross, A primer on Pontryagin's principle in optimal control, CA: Collegiate Publishers, 2015.

[25] T. R. Jorris, R. G. Cobb, Multiple method 2-D trajectory optimization satisfying waypoints and no-fly zone constraints, Journal of Guidance, Control, and Dynamics 31 (3) (2008) 543-553. doi:10.2514/1.32354.

[26] T. R. Jorris, R. G. Cobb, Three-dimensional trajectory optimization satisfying waypoint and no-fly zone constraints, Journal of Guidance, Control, and Dynamics 32 (2) (2009) 551-572. doi:10.2514/1.37030.

1025

[27] A. Weiss, M. Baldwin, R. S. Erwin, I. Kolmanovsky, Model predictive control for spacecraft rendezvous and docking: Strategies for handling constraints and case studies, IEEE Transactions on Control Systems Technology 23 (4) (2015) 1638-1647. doi:10.1109/TCST.2014.2379639. 
[28] H. Zhang, X. Zhang, Pointwise second-order necessary conditions for stochastic optimal controls, part i: The case of convex control constraint, SIAM Journal on Control and Optimization 53 (4) (2015) 2267-2296. doi : 10.1137/14098627X.

[29] A. Boccia, M. de Pinho, R. Vinter, Optimal control problems with mixed and pure state constraints, SIAM Journal on Control and Optimization 54 (6) (2016) 3061-3083. doi:10.1137/15M1041845.

[30] J. Bonnans, A. Festa, Error estimates for the euler discretization of an optimal control problem with first-order state constraints, SIAM Journal on Numerical Analysis 55 (2) (2017) 445-471. doi:10.1137/140999621.

[31] W. w. Cai, Y. w. Zhu, L. p. Yang, Y. w. Zhang, Optimal guidance for hypersonic reentry using inversion and receding horizon control, IET Control Theory and Applications 9 (9) (2015) 1347-1355. doi:10.1049/iet-cta. 2014.1155.

[32] C.-F. Hu, Y. Xin, Reentry trajectory optimization for hypersonic vehicles using fuzzy satisfactory goal programming method, International Journal of Automation and Computing 12 (2) (2015) 171-181.

[33] R. Chai, A. Savvaris, A. Tsourdos, Fuzzy physical programming for space manoeuvre vehicles trajectory optimization based on hp-adaptive pseudospectral method, Acta Astronautica 123 (2016) 62-70. doi:http: //dx.doi.org/10.1016/j.actaastro.2016.02.020.

[34] J. A. Englander, B. A. Conway, Automated solution of the low-thrust interplanetary trajectory problem, Journal of Guidance, Control, and Dynamics 40 (1) (2017) 15-27. doi:10.2514/1.G002124.

[35] M. Pontani, B. A. Conway, Particle swarm optimization applied to space trajectories, Journal of Guidance, Control, and Dynamics 33 (5) (2010) 1429-1441. doi:10.2514/1.48475.

[36] M. Pontani, B. A. Conway, Particle swarm optimization applied to impulsive orbital transfers, Acta Astronautica 74 (2012) 141-155. doi:https: //doi.org/10.1016/j.actaastro.2011.09.007. 
[37] G. Ambrosino, M. Ariola, U. Ciniglio, F. Corraro, E. D. Lellis, A. Pironti,

Path generation and tracking in 3-d for uavs, IEEE Transactions on Control Systems Technology 17 (4) (2009) 980-988. doi:10.1109/TCST . 2009. 2014359.

[38] Y. Wang, S. Wang, M. Tan, C. Zhou, Q. Wei, Real-time dynamic dubinshelix method for 3-d trajectory smoothing, IEEE Transactions on Control Systems Technology 23 (2) (2015) 730-736. doi:10.1109/TCST.2014. 2325904.

[39] X. Ma, Z. Jiao, Z. Wang, D. Panagou, 3-d decentralized prioritized motion planning and coordination for high-density operations of micro aerial vehicles, IEEE Transactions on Control Systems Technology 26 (3) (2018) 939-953. doi:10.1109/TCST.2017.2699165.

[40] J. Nocedal, S. J. Wright, Numerical Optimization, Springer series in operations research, 2006.

[41] J. Laurent-Varin, F. Bonnans, N. Berend, M. Haddou, C. Talbot, Interiorpoint approach to trajectory optimization, Journal of Guidance, Control, and Dynamics 30 (5) (2007) 1228-1238. doi:10.2514/1.18196.

[42] R. Chai, A. Savvaris, A. Tsourdos, S. Chai, Y. Xia, Improved gradientbased algorithm for solving aeroassisted vehicle trajectory optimization problems, Journal of Guidance, Control, and Dynamics 40 (8) (2017) 2093-2101. doi:10.2514/1.G002183.

[43] X. Liu, P. Lu, B. Pan, Survey of convex optimization for aerospace applications, Astrodynamics 1 (1) (2017) 23-40. doi:10.1007/ s42064-017-0003-8.

[44] X. Liu, Z. Shen, P. Lu, Entry trajectory optimization by second-order cone programming, Journal of Guidance, Control, and Dynamics 39 (2) (2016) 227-241. doi:10.2514/1.G001210.

[45] J. Helton, J. Nie, Sufficient and necessary conditions for semidefinite representability of convex hulls and sets, SIAM Journal on Optimization 20 (2) (2009) 759-791. doi:10.1137/07070526X. 
[46] W. Hongying, C. Nayibe Chio, H. Bouadi, Z. Lunlong, F. Mora-Camino, Dynamic programming for trajectory optimization of engine-out transportation aircraft, in: 2012 24th Chinese Control and Decision Conference (CCDC), 2012, pp. 98-103. doi:10.1109/CCDC.2012.6244015.

[47] J. D. Aziz, J. S. Parker, D. J. Scheeres, J. A. Englander, Low-thrust manyrevolution trajectory optimization via differential dynamic programming and a sundman transformation, The Journal of the Astronautical Sciences 65 (2) (2018) 205-228. doi:10.1007/s40295-017-0122-8.

[48] N. Ozaki, S. Campagnola, R. Funase, C. H. Yam, Stochastic differential dynamic programming with unscented transform for low-thrust trajectory design, Journal of Guidance, Control, and Dynamics 41 (2) (2017) 377387. doi:10.2514/1.G002367.

[49] S. M. Elsayed, R. A. Sarker, D. L. Essam, An improved self-adaptive differential evolution algorithm for optimization problems, IEEE Transactions on Industrial Informatics 9 (1) (2013) 89-99. doi:10.1109/TII.2012. 2198658.

[50] R. Chai, A. Savvaris, A. Tsourdos, Violation learning differential evolution-based hp-adaptive pseudospectral method for trajectory optimization of space maneuver vehicle, IEEE Transactions on Aerospace and Electronic Systems 53 (4) (2017) 2031-2044. doi:10.1109/TAES. 2017. 2680698.

[51] B. Zhang, H. Duan, Three-dimensional path planning for uninhabited combat aerial vehicle based on predator-prey pigeon-inspired optimization in dynamic environment, IEEE/ACM Transactions on Computational Biology and Bioinformatics 14 (1) (2017) 97-107. doi :10.1109/TCBB . 2015. 2443789.

[52] G. Radice, G. Olmo, Ant colony algorithms for two impluse interplanetary trajectory optimization, Journal of Guidance, Control, and Dynamics 29 (6) (2006) 1440-1444. doi:10.2514/1.20828.

[53] H. Duan, S. Li, Artificial bee colony based direct collocation for reentry trajectory optimization of hypersonic vehicle, IEEE Transactions on 
Aerospace and Electronic Systems 51 (1) (2015) 615-626. doi:10.1109/ TAES. 2014.120654.

[54] P. Lu, M. A. Khan, Nonsmooth trajectory optimization - an approach using continuous simulated annealing, Journal of Guidance, Control, and Dynamics 17 (4) (1994) 685-691. doi:10.2514/3.21256.

[55] H. Nobahari, A. Haeri, A heuristic predictive los guidance law based on trajectory learning, ant colony optimization and tabu search, in: 2016 6th IEEE International Conference on Control System, Computing and Engineering (ICCSCE), 2016, pp. 163-168. doi:10.1109/ICCSCE. 2016. 7893564.

[56] M. Heinkenschloss, D. Ridzal, A matrix-free trust-region sqp method for equality constrained optimization, SIAM Journal on Optimization 24 (3) (2014) 1507-1541. doi:doi:10.1137/130921738.

[57] B. Senses, A. V. Rao, Optimal finite-thrust small spacecraft aeroassisted orbital transfer, Journal of Guidance, Control, and Dynamics 36 (6) (2013) 1802-1810. doi:10.2514/1.58977.

[58] J. J. Kim, J. J. Lee, Trajectory optimization with particle swarm optimization for manipulator motion planning, IEEE Transactions on Industrial Informatics 11 (3) (2015) 620-631. doi:10.1109/TII.2015.2416435.

[59] N. Yokoyama, S. Suzuki, Modified genetic algorithm for constrained trajectory optimization, Journal of Guidance, Control, and Dynamics 28 (1) (2005) 139-144. doi:10.2514/1.3042.

[60] K. Subbarao, B. M. Shippey, Hybrid genetic algorithm collocation method for trajectory optimization, Journal of Guidance, Control, and Dynamics 32 (4) (2009) 1396-1403. doi:10.2514/1.41449.

[61] M. Ceriotti, M. Vasile, Mga trajectory planning with an aco-inspired algorithm, Acta Astronautica 67 (9) (2010) 1202-1217. doi:https: //doi.org/10.1016/j.actaastro.2010.07.001.

[62] J. A. Englander, B. A. Conway, Automated solution of the low-thrust interplanetary trajectory problem, Journal of Guidance, Control, and Dynamics 40 (1) (2016) 15-27. doi:10.2514/1.G002124. 
[63] P. C. Roy, M. M. Islam, K. Murase, X. Yao, Evolutionary path control strategy for solving many-objective optimization problem, IEEE Transactions on Cybernetics 45 (4) (2015) 702-715. doi:10.1109/TCYB. 2014. 2334632.

[64] X. Liu, P. Lu, Solving nonconvex optimal control problems by convex optimization, Journal of Guidance, Control, and Dynamics 37 (3) (2014) 750-765. doi:10.2514/1.62110.

[65] B. Acikmese, J. M. Carson, L. Blackmore, Lossless convexification of nonconvex control bound and pointing constraints of the soft landing optimal control problem, IEEE Transactions on Control Systems Technology 21 (6) (2013) 2104-2113. doi:10.1109/TCST . 2012.2237346.

[66] M. W. Harris, B. Acikmese, Maximum divert for planetary landing using convex optimization, Journal of Optimization Theory and Applications 162 (3) (2014) 975-995. doi:10.1007/s10957-013-0501-7.

[67] J. B. Mueller, P. R. Griesemer, S. J. Thomas, Avoidance maneuver planning incorporating station-keeping constraints and automatic relaxation, Journal of Aerospace Information Systems 10 (6) (2013) 306-322. doi:10.2514/1.54971.

[68] C. Colombo, M. Vasile, G. Radice, Optimal low-thrust trajectories to asteroids through an algorithm based on differential dynamic programming, Celestial Mechanics and Dynamical Astronomy 105 (1) (2009) 75. doi:10.1007/s10569-009-9224-3.

[69] G. Lantoine, R. P. Russell, A hybrid differential dynamic programming algorithm for constrained optimal control problems. part 1: Theory, Journal of Optimization Theory and Applications 154 (2) (2012) 382-417. doi:10.1007/s10957-012-0039-0.

[70] G. Lantoine, R. P. Russell, A hybrid differential dynamic programming algorithm for constrained optimal control problems. part 2: Application, Journal of Optimization Theory and Applications 154 (2) (2012) 418-442. doi:10.1007/s10957-012-0038-1. 
[71] C.-H. Huang, J. Galuski, C. L. Bloebaum, Multi-objective pareto concurrent subspace optimization for multidisciplinary design, AIAA Journal 45 (8) (2007) 1894-1906. doi:10.2514/1.19972.

[72] R. Azizipanah-Abarghooee, V. Terzija, F. Golestaneh, A. Roosta, Multiobjective dynamic optimal power flow considering fuzzy-based smart utilization of mobile electric vehicles, IEEE Transactions on Industrial Informatics 12 (2) (2016) 503-514. doi:10.1109/TII.2016.2518484.

[73] Y. Shen, Y. Wang, Operating point optimization of auxiliary power unit using adaptive multi-objective differential evolution algorithm, IEEE Transactions on Industrial Electronics 64 (1) (2017) 115-124. doi: 10.1109/TIE. 2016.2598674.

[74] R. Chai, A. Savvaris, A. Tsourdos, S. Chai, Solving Multi-objective Aeroassisted Spacecraft Trajectory Optimization Problems Using Extended NSGA-II, AIAA SPACE Forum, American Institute of Aeronautics and Astronautics, 2017. doi:10.2514/6.2017-5193.

[75] R. Chai, A. Savvaris, A. Tsourdos, S. Chai, Y. Xia, Unified multiobjective optimization scheme for aeroassisted vehicle trajectory planning, Journal of Guidance, Control, and Dynamics 41 (7) (2018) 1521-1530. doi:10. 2514/1.G003189.

[76] R. Chai, A. Savvaris, A. Tsourdos, Y. Xia, S. Chai, Solving multiobjective constrained trajectory optimization problem by an extended evolutionary algorithm, IEEE Transactions on Cybernetics (2018) 1-14doi:10.1109/ TCYB. 2018.2881190.

[77] S. Jiang, S. Yang, Evolutionary dynamic multiobjective optimization: Benchmarks and algorithm comparisons, IEEE Transactions on Cybernetics 47 (1) (2017) 198-211. doi:10.1109/TCYB.2015.2510698.

[78] B. Xue, M. Zhang, W. N. Browne, Particle swarm optimization for feature selection in classification: A multi-objective approach, IEEE Transactions on Cybernetics 43 (6) (2013) 1656-1671. doi:10.1109/TSMCB. 2012.2227469 . 
[79] H. Han, W. Lu, J. Qiao, An adaptive multiobjective particle swarm optimization based on multiple adaptive methods, IEEE Transactions on Cybernetics 47 (9) (2017) 2754-2767. doi:10.1109/TCYB. 2017.2692385.

[80] H. Han, X. Wu, L. Zhang, Y. Tian, J. Qiao, Self-organizing rbf neural network using an adaptive gradient multiobjective particle swarm optimization, IEEE Transactions on Cybernetics (2017) 1-14doi:10.1109/ TCYB. 2017.2764744.

[81] R. Akbari, R. Hedayatzadeh, K. Ziarati, B. Hassanizadeh, A multiobjective artificial bee colony algorithm, Swarm and Evolutionary Computation 2 (2012) 39-52. doi:https://doi.org/10.1016/j.swevo. 2011. 08.001 .

[82] M. A. Abido, A niched pareto genetic algorithm for multiobjective environmental/economic dispatch, International Journal of Electrical Power \& Energy Systems 25 (2) (2003) 97-105. doi:http://dx.doi.org/10. 1016/S0142-0615(02)00027-3.

[83] A. Elhossini, S. Areibi, R. Dony, Strength pareto particle swarm optimization and hybrid ea-pso for multi-objective optimization, Evolutionary Computation 18 (1) (2010) 127-156. doi:10.1162/evco.2010.18.1. 18105.

[84] R. Chai, A. Savvaris, A. Tsourdos, S. Chai, Multi-objective trajectory optimization of space manoeuvre vehicle using adaptive differential evolution and modified game theory, Acta Astronautica 136 (2017) 273-280. doi:http://dx.doi.org/10.1016/j.actaastro.2017.02.023.

[85] H.-G. Han, H.-H. Qian, J.-F. Qiao, Nonlinear multiobjective modelpredictive control scheme for wastewater treatment process, Journal of Process Control 24 (3) (2014) 47-59. doi:https://doi.org/10.1016/j . jprocont.2013.12.010.

[86] A. Messac, Physical programming - effective optimization for computational design, AIAA Journal 34 (1) (1996) 149-158. doi:10.2514/3. 13035 .

[87] R. V. Tappeta, J. E. Renaud, A. Messac, G. J. Sundararaj, Interactive physical programming: Tradeoff analysis and decision making in 
multicriteria optimization, AIAA Journal 38 (5) (2000) 917-926. doi: $10.2514 / 2.1048$.

[88] R. Chai, A. Savvaris, A. Tsourdos, Y. Xia, An interactive fuzzy physical programming for skip entry problem, IEEE Transactions on Aerospace and Electronic Systems 53 (5) (2017) 2385-2398. doi:10.1109/TAES. 2017.2696281.

[89] L.-H. Chen, F.-C. Tsai, Fuzzy goal programming with different importance and priorities, European Journal of Operational Research 133 (3) (2001) 548-556. doi:http://dx.doi.org/10.1016/S0377-2217(00)00201-0.

[90] W. Wang, H. Peng, A fast multi-objective optimization design method for emergency libration point orbits transfer between the sun-earth and the earth-moon systems, Aerospace Science and Technology 63 (2017) 152166. doi:https://doi.org/10.1016/j.ast.2016.12.026.

[91] C. Dai, Y. Wang, M. Ye, A new multi-objective particle swarm optimization algorithm based on decomposition, Information Sciences 325 (Supplement C) (2015) 541-557. doi:https://doi.org/10.1016/j.ins. 2015. 07.018.

[92] I. Giagkiozis, P. J. Fleming, Methods for multi-objective optimization: An analysis, Information Sciences 293 (Supplement C) (2015) 338-350. doi:https://doi.org/10.1016/j.ins.2014.08.071.

[93] J. Cheng, G. G. Yen, G. Zhang, A grid-based adaptive multi-objective differential evolution algorithm, Information Sciences 367 (Supplement C) (2016) 890-908. doi:https://doi.org/10.1016/j.ins.2016.07.009.

[94] S. Lalwani, S. Singhal, R. Kumar, N. Gupta, A comprehensive survey: Applications of multi-objective particle swarm optimization (mopso) algorithm, Transactions on Combinatorics 2 (1) (2013) 39-101.

[95] M. Vasile, F. Zuiani, A hybrid multiobjective optimization algorithm applied to space trajectory optimization, 2010, pp. 1-8. doi:10.1109/CEC. 2010.5586240 .

[96] B. Xue, M. Zhang, W. N. Browne, Particle swarm optimization for feature selection in classification: A multi-objective approach, IEEE Trans- 
actions on Cybernetics 43 (6) (2013) 1656-1671. doi:10.1109/TSMCB.

2012.2227469 .

[97] V. A. Kostenko, A. V. Frolov, Self-learning genetic algorithm, Journal of Computer and Systems Sciences International 54 (4) (2015) 525-539. doi:10.1134/s1064230715040103.

[98] Y. J. Gong, J. J. Li, Y. Zhou, Y. Li, H. S. H. Chung, Y. H. Shi, J. Zhang, Genetic learning particle swarm optimization, IEEE Transactions on Cybernetics 46 (10) (2016) 2277-2290. doi:10.1109/TCYB.2015.2475174.

[99] W. Hu, G. G. Yen, G. Luo, Many-objective particle swarm optimization using two-stage strategy and parallel cell coordinate system, IEEE Transactions on Cybernetics 47 (6) (2017) 1446-1459. doi:10.1109/TCYB.2016. 2548239.

[100] B. Ji, X. Yuan, Y. Yuan, Modified NSGA-II for solving continuous berth allocation problem: Using multiobjective constraint-handling strategy, IEEE Transactions on Cybernetics 47 (9) (2017) 2885-2895. doi:10. 1109/TCYB. 2017.2669334.

[101] X. Cai, Z. Yang, Z. Fan, Q. Zhang, Decomposition-based-sorting and angle-based-selection for evolutionary multiobjective and many-objective optimization, IEEE Transactions on Cybernetics 47 (9) (2017) 2824-2837. doi:10.1109/TCYB.2016.2586191.

[102] O. Schutze, M. Vasile, O. Junge, M. Dellnitz, D. Izzo, Designing optimal low-thrust gravity-assist trajectories using space pruning and a multi-objective approach, Engineering Optimization 41 (2) (2009) 155181. doi:10.1080/03052150802391734.

[103] M. Martens, D. Izzo, The asynchronous island model and nsga-ii: Study of a new migration operator and its performance, in: Proceedings of the 15th Annual Conference on Genetic and Evolutionary Computation, GECCO '13, ACM, New York, NY, USA, 2013, pp. 1173-1180. doi:10.1145/ 2463372.2463516 .

[104] P. A. N. Bosman, On gradients and hybrid evolutionary algorithms for real-valued multiobjective optimization, IEEE Transactions on Evolutionary Computation 16 (1) (2012) 51-69. doi :10.1109/TEVC. 2010. 2051445. 
[105] B. Chen, W. Zeng, Y. Lin, D. Zhang, A new local search-based multiobjective optimization algorithm, IEEE Transactions on Evolutionary Computation 19 (1) (2015) 50-73. doi:10.1109/TEVC.2014.2301794.

[106] K. Deb, H. Jain, An evolutionary many-objective optimization algorithm using reference-point-based nondominated sorting approach, part i: Solving problems with box constraints, IEEE Transactions on Evolutionary Computation 18 (4) (2014) 577-601. doi:10.1109/TEVC.2013.2281535.

[107] D. Gonzalez-Arribas, M. Soler, M. Sanjurjo-Rivo, Robust aircraft trajectory planning under wind uncertainty using optimal control, Journal of Guidance, Control, and Dynamics (2017) 1-16doi:10.2514/1.G002928.

[108] Z. Zhao, M. Kumar, Split-bernstein approach to chance-constrained optimal control, Journal of Guidance, Control, and Dynamics 40 (11) (2017) 2782-2795. doi:10.2514/1.G002551.

[109] L. Blackmore, M. Ono, B. C. Williams, Chance-constrained optimal path planning with obstacles, IEEE Transactions on Robotics 27 (6) (2011) 1080-1094. doi:10.1109/TRO.2011.2161160.

[110] M. Cannon, Q. Cheng, B. Kouvaritakis, S. V. Rakovic, Stochastic tube mpc with state estimation, Automatica 48 (3) (2012) 536-541. doi: https://doi.org/10.1016/j . automatica.2011.08.058.

[111] M. Cannon, B. Kouvaritakis, S. V. Rakovic, Q. Cheng, Stochastic tubes in model predictive control with probabilistic constraints, IEEE Transactions on Automatic Control 56 (1) (2011) 194-200. doi:10.1109/TAC. 2010. 2086553.

[112] S. Salomon, G. Avigad, P. J. Fleming, R. C. Purshouse, Active robust optimization: Enhancing robustness to uncertain environments, IEEE Transactions on Cybernetics 44 (11) (2014) 2221-2231. doi:10.1109/TCYB. 2014.2304475 .

[113] H. Gui, G. Vukovich, S. Xu, Attitude tracking of a rigid spacecraft using two internal torques, IEEE Transactions on Aerospace and Electronic Systems 51 (4) (2015) 2900-2913. doi:10.1109/TAES.2015.140670. 
[114] L. Wang, Y. Xia, Mars entry navigation with uncertain parameters based on desensitized extended kalman filter, IEEE Transactions on Industrial Informatics 11 (5) (2015) 998-1005. doi:10.1109/TII.2015.2463763.

[115] T. Chan, P. Mar, Stability and continuity in robust optimization, SIAM Journal on Optimization 27 (2) (2017) 817-841. doi:10.1137/ 16 M1067512.

[116] H. Li, Y. Shi, Robust distributed model predictive control of constrained continuous-time nonlinear systems: A robustness constraint approach, IEEE Transactions on Automatic Control 59 (6) (2014) 1673-1678. doi: 10.1109/TAC. 2013. 2294618.

[117] X. Qiu, J. X. Xu, Y. Xu, K. C. Tan, A new differential evolution algorithm for minimax optimization in robust design, IEEE Transactions on Cybernetics PP (99) (2017) 1-14. doi:10.1109/TCYB.2017.2692963.

[118] S. Wang, W. Pedrycz, Data-driven adaptive probabilistic robust optimization using information granulation, IEEE Transactions on Cybernetics 48 (2) (2018) 450-462. doi:10.1109/TCYB.2016.2638461.

[119] D. Bienstock, M. Chertkov, S. Harnett, Chance-constrained optimal power flow: Risk-aware network control under uncertainty, SIAM Review 56 (3) (2014) 461-495. doi:10.1137/130910312.

[120] N. Wan, C. Zhang, A. Vahidi, Probabilistic anticipation and control in autonomous car following, IEEE Transactions on Control Systems Technology 27 (1) (2019) 30-38. doi:10.1109/TCST.2017.2762288.

[121] M. P. Vitus, Z. Zhou, C. J. Tomlin, Stochastic control with uncertain parameters via chance constrained control, IEEE Transactions on Automatic Control 61 (10) (2016) 2892-2905. doi:10.1109/TAC.2015.2511587.

[122] A. Nemirovski, A. Shapiro, Convex approximations of chance constrained programs, SIAM Journal on Optimization 17 (4) (2006) 969-996. doi: 10.1137/050622328.

[123] A. Geletu, M. Kloppel, A. Hoffmann, P. Li, A tractable approximation of non-convex chance constrained optimization with non-gaussian 
uncertainties, Engineering Optimization 47 (4) (2015) 495-520. doi: 10.1080/0305215X.2014.905550.

[124] A. Geletu, A. Hoffmann, M. Kloppel, P. Li, An inner-outer approximation approach to chance constrained optimization, SIAM Journal on Optimization 27 (3) (2017) 1834-1857. doi:10.1137/15M1049750.

[125] M. Lorenzen, F. Dabbene, R. Tempo, F. Allgower, Constraint-tightening and stability in stochastic model predictive control, IEEE Transactions on Automatic Control 62 (7) (2017) 3165-3177. doi:10.1109/TAC. 2016. 2625048.

[126] G. C. Calafiore, L. Fagiano, Robust model predictive control via scenario optimization, IEEE Transactions on Automatic Control 58 (1) (2013) 219224. doi:10.1109/TAC.2012.2203054.

[127] A. E.-M. A. Mohamed, M. A. A. El-Hadidy, Coordinated search for a conditionally deterministic target motion in the plane, European Journal of Mathematical Sciences 2 (3) (2013) 272-295.

[128] T. Huschto, S. Sager, Solving stochastic optimal control problems by a wiener chaos approach, Vietnam Journal of Mathematics 42 (1) (2014) 83-113. doi:10.1007/s10013-014-0060-8.

[129] P. Dutta, R. Bhattacharya, Nonlinear estimation of hypersonic state trajectories in bayesian framework with polynomial chaos, Journal of Guidance, Control, and Dynamics 33 (6) (2010) 1765-1778. doi:10.2514/1. 49743.

[130] C. Chawla, P. Sarmah, R. Padhi, Suboptimal reentry guidance of a reusable launch vehicle using pitch plane maneuver, Aerospace Science and Technology 14 (6) (2010) 377-386. doi:http://dx.doi.org/10.1016/j . ast.2010.04.001.

[131] B. Tian, Q. Zong, Optimal guidance for reentry vehicles based on indirect legendre pseudospectral method, Acta Astronautica 68 (7) (2011) 11761184. doi:http://dx.doi.org/10.1016/j.actaastro.2010.10.010.

[132] Y. Xia, R. Chen, F. Pu, L. Dai, Active disturbance rejection control for drag tracking in mars entry guidance, Advances in Space Research 53 
(5) (2014) 853-861. doi:http://dx.doi.org/10.1016/j.asr.2013.12. 008.

[133] Y.Xia, G. Shen, L. Zhou, H. Sun, Mars entry guidance based on segmented guidance predictor-corrector algorithm, Control Engineering Practice 45 (2015) 79-85. doi:http://dx.doi.org/10.1016/j.conengprac.2015. 08.006 .

[134] J. Dai, Y. Xia, Mars atmospheric entry guidance for reference trajectory tracking, Aerospace Science and Technology 45 (2015) 335-345. doi: http://dx.doi.org/10.1016/j.ast.2015.06.006.

[135] B. Kevin, R. Michael, D. David, Optimal Nonlinear Feedback Guidance for Reentry Vehicles, Guidance, Navigation, and Control and Co-located Conferences, American Institute of Aeronautics and Astronautics, 2006. doi:doi:10.2514/6.2006-6074.

[136] B. Panchal, N. Mate, S. E. Talole, Continuous-time predictive controlbased integrated guidance and control, Journal of Guidance, Control, and Dynamics (2017) 1-17.

[137] X. Liu, F. Zhang, Z. Li, Y. Zhao, Approach and landing guidance design for reusable launch vehicle using multiple sliding surfaces technique, Chinese Journal of Aeronautics 30 (4) (2017) 1582-1591. doi:https://doi.org/ $10.1016 / j . c j a .2017 .06 .008$.

[138] X. Liu, K. D. Kumar, Network-based tracking control of spacecraft formation flying with communication delays, IEEE Transactions on Aerospace and Electronic Systems 48 (3) (2012) 2302-2314. doi:10.1109/TAES. 2012.6237593.

[139] G. Liu, S. Zhang, A survey on formation control of small satellites, Proceedings of the IEEE 106 (3) (2018) 440-457. doi:10.1109/JPROC. 2018. 2794879.

[140] R. Kristiansen, P. J. Nicklasson, Spacecraft formation flying: A review 1425 and new results on state feedback control, Acta Astronautica 65 (11) (2009) 1537-1552. doi:https://doi.org/10.1016/j.actaastro. 2009. 04.014. 
[141] L. Pettazzi, H. Kruger, S. Theil, D. Izzo, Electrostatic force for swarm navigation and reconfiguration, Acta Futura 4 (2008) 80-86.

${ }_{1430}[142]$ N. Petit, M. Milam, R. Murray, Constrained trajectory generation for micro-satellite formation flying, Guidance, Navigation, and Control and Co-located Conferences, American Institute of Aeronautics and Astronautics, 2001. doi:doi:10.2514/6.2001-4030.

[143] Y. Kim, M. Mesbahi, F. Y. Hadaegh, Dual-spacecraft formation flying in deep space: Optimal collision-free reconfigurations, Journal of Guidance, Control, and Dynamics 26 (2) (2003) 375-379. doi:10.2514/2.5059.

[144] M. Sagliano, E. Mooij, S. Theil, Onboard Trajectory Generation for Entry Vehicles via Adaptive Multivariate Pseudospectral Interpolation, AIAA SciTech Forum, American Institute of Aeronautics and Astronautics, 2016, doi:10.2514/6.2016-2115. doi:doi:10.2514/6.2016-2115.

[145] M. Sagliano, E. Mooij, S. Theil, Onboard trajectory generation for entry vehicles via adaptive multivariate pseudospectral interpolation, Journal of Guidance, Control, and Dynamics 40 (2) (2017) 466-476. doi:10.2514/ 1.G001817. 
2019-06-04

\section{A review of optimization techniques in spacecraft flight trajectory design}

Chai, Runqi

Elsevier

Chai R, Savvaris A, Tsourdos A, et al., (2019) A review of optimization techniques in spacecraft flight trajectory design. Progress in Aerospace Sciences, Volume 109, August 2019, Article number 100543

https://doi.org/10.1016/j.paerosci.2019.05.003

Downloaded from Cranfield Library Services E-Repository 